\title{
Theta-burst transcranial magnetic stimulation for the treatment of unilateral neglect in stroke patients: A systematic review and best evidence synthesis
}

\author{
Milan Houben ${ }^{\mathrm{a}}$, Sabrina Chettouf ${ }^{\mathrm{a}}$, Ysbrand D. Van Der Werf ${ }^{\mathrm{b}}$ and John Stins ${ }^{\mathrm{a}, *}$ \\ ${ }^{a}$ Department of Human Movement Sciences, Faculty of Behavioural and Movement Sciences, Vrije Universiteit \\ Amsterdam, Amsterdam Movement Sciences, Van der Boechorststraat 7 Amsterdam, The Netherlands \\ ${ }^{\mathrm{b}}$ Department of Anatomy and Neurosciences, Amsterdam UMC, Vrije Universiteit Amsterdam, Amsterdam \\ Neuroscience, De Boelelaan 1117 Amsterdam, The Netherlands
}

\begin{abstract}
.
Background: Unilateral neglect (UN) is a common and disabling disorder after stroke. UN is a strong and negative predictor of functional rehabilitative outcome. Non-invasive brain stimulation, such as theta-burst transcranial magnetic stimulation (TBS), is a promising rehabilitation technique for treating stroke-induced UN.

Objective: To systematically review the available literature, researching whether TBS of the contra-lesional hemisphere is more effective than standard rehabilitation in improving symptoms of UN in patients with right hemisphere stroke.

Review methods: A systematic review was conducted to retrieve randomized controlled trials (RCTs) that were relevant to the objective of this review. PubMed, Ovid and Cochrane Library electronic databases were comprehensively searched from inception up to February 2021. Of the included studies, methodological quality was assessed using the PEDro scale, whereafter a best evidence synthesis (BES) was conducted to summarize the results.

Results: Nine RCTs investigating the effects of TBS on stroke-induced UN symptoms were included in this review. Seven studies assessing continuous TBS (cTBS) found significantly greater amelioration of UN symptoms in the TBS intervention group when compared to the control group; one study assessing cTBS found no such significant difference. One study assessing intermittent TBS (iTBS) found significant between-group differences in favor of the intervention. The BES yielded strong evidence in favor of cTBS, and limited evidence in favor of iTBS.

Conclusions: The included studies in the present review allow the conclusion that TBS can have favorable effects on UN recovery in stroke patients. Its clinical use is recommended in conjunction with cognitive rehabilitation and occupational or physical rehabilitation as needed. However, many aspects for optimal usage of TBS therapy in clinical settings, such as exact TBS protocols, number of sessions, and treatment duration, are not clear.
\end{abstract}

Keywords: Transcranial magnetic stimulation, theta-burst stimulation, unilateral neglect, stroke, systematic review

\section{Introduction}

Stroke is the second most common cause of death and the leading cause of chronic adult disability

${ }^{*}$ Corresponding author: Dr. John Stins, Van der Boechorststraat 7 Amsterdam, Amsterdam, 1081 HV, The Netherlands. Tel.: +31 (0)20 598 8543; E-mail: j.f.stins@vu.nl. in Europe (Wafa et al., 2020). In 2017, there were approximately 1.12 million strokes and 9.53 million individuals living with the consequences of stroke in 
Europe alone (Wafa et al., 2020). Stroke is a disabling condition with a considerable socioeconomic impact due to high health-care costs and loss of labour productivity (Feigin et al., 2016; Maaijwee et al., 2014). The absolute number of strokes has been increasing and is expected to keep doing so over the next 30 years (Wafa et al., 2020). The increase in stroke survivors, and the associated costs, makes cost-effective treatment of the utmost importance. To date, no treatments can restore function through repair of damaged brain tissue. Current best-practice stroke management consists mostly of reduction of initial impact, secondary stroke prevention, precautions to avoid future complications, and maximisation of functional ability (Campbell \& Khatri, 2020).

Among stroke survivors, unilateral neglect (UN), also commonly known as hemispatial neglect or hemineglect, is a frequently occurring and disabling condition. UN is a perceptual-attentional disorder characterized by a reduction or loss of spatial awareness for the contralesional space (Buxbaum et al., 2004). Stroke patients with UN have longer rehabilitation hospitalizations, have inferior rehabilitation outcome of other stroke symptoms, and are more impaired in activities of daily living (ADL) than stroke patients without UN (Buxbaum et al., 2004; Di Monaco et al., 2011; Gillen et al., 2005; Stone et al., 1992). In a recent meta-analysis, estimated prevalence of UN after unihemispheric stroke was 38\% after right-side brain damage and $18 \%$ after left-side brain damage (Esposito et al., 2021). Several theoretical and anatomical models have been proposed to account for the higher prevalence of UN after right hemisphere damage, as compared to left hemisphere damage.

\subsection{Diagnosis of neglect and measures of its symptoms}

Disorders such as unilateral neglect can be classified within WHO's international classification of function, disability, and health (ICF-model; Fig. 1A). This system provides a framework in which the effects of a certain disorder on an individual's health are classified in terms of pathology (underlying disease or disorder), impairments of function, limitations in activity, and restrictions in participation (henceforth collectively called 'health aspects'; Fig. 1B) (Ustün et al., 2003). UN presents itself with a large variability in symptoms (Buxbaum et al., 2004), affecting all the aforementioned health aspects. Standardized outcome measures are available for all the different health aspects classified by the ICFmodel (Fig. 1C) (Langhorne et al., 2011). These outcome measures of neglect severity and accompanying impairments, disabilities and handicaps can provide information about treatment requirements and eventual treatment effectiveness. These measures can therefore guide neurorehabilitative efforts.

\subsection{Treatment of neglect}

Approximately two thirds of the neglect patients seem to recover from stroke-induced UN symptoms in the sub-acute phase, leaving one third of the patients who still show clear signs of neglect 1 year after stroke onset (Karnath et al., 2011). Neurorehabilitative techniques focus on functional recovery and can broadly be classified as: (1) retraining functionality (restitution); (2) utilization of remaining intact brain structures and functions (compensation); and (3) adaptation to functional impairments by the usage of external devices or modifications (substitution) (Ting et al., 2011).

Over the years, many different cognitive rehabilitation interventions have been developed for UN treatment (Kerkhoff, 2001; Ting et al., 2011). A Cochrane review assessing the effectiveness of cognitive rehabilitation in $23 \mathrm{RCTs}$, concluded that there is insufficient evidence to support or refute the effectiveness of cognitive rehabilitation on neglect symptoms (Bowen et al., 2013). Several recently developed and promising rehabilitation techniques were, however, not included in this review, like noninvasive brain stimulation (NIBS). NIBS, such as repetitive transcranial magnetic stimulation (rTMS) and transcranial direct current stimulation (tDCS), can be used to modulate the excitability of neuronal cortical circuits (Di Pino et al., 2014), with the aim to ameliorate symptoms after stroke, such as neglect. Neuromodulatory techniques like NIBS can be broadly classified into protocols that have an excitatory effect (long-term potentiation-like activity), and protocols that have an inhibitory effect (longterm depression-like activity) on cortical excitability (Di Pino et al., 2014). Excitatory NIBS protocols include anodal tDCS, high-frequency rTMS (HF rTMS), and intermittent theta-burst stimulation (iTBS). Inhibiting protocols include cathodal tDCS, low-frequency rTMS (LF rTMS) and continuous theta-burst stimulation (cTBS) (Di Pino et al., 2014).

The rationale behind using NIBS in UN treatment is based on various models of neglect, such as the interhemispheric rivalry model, originally 
A

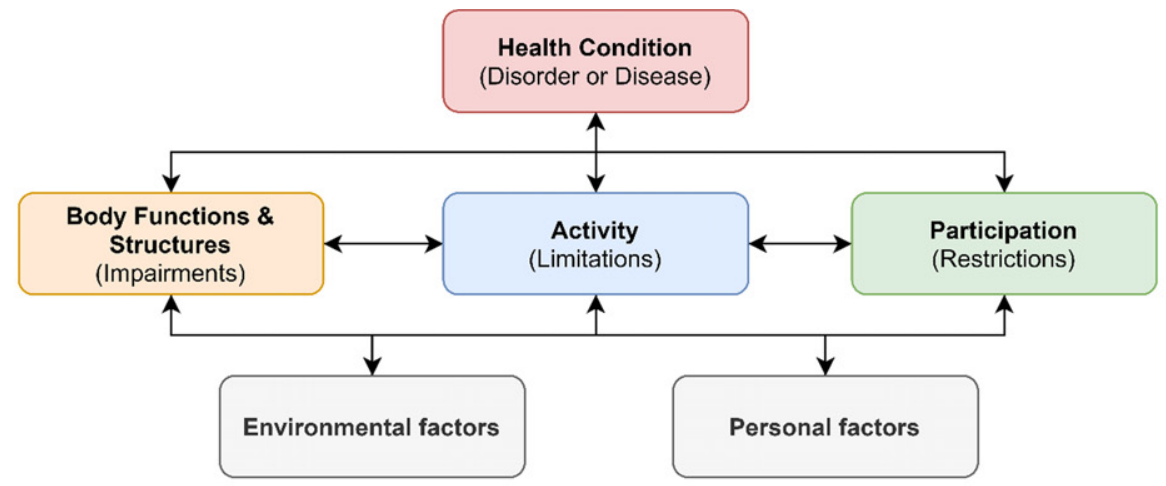

B

Relevant body functions affected:
- Attention networks
- Spatial awareness
- Attentional orienting
- Attentional selection
Relevant body structures affected:
- Brain

Common types of Unilateral Neglect: - Sensory Neglect (e.g. visuospatial)

- Motor or Premotor Neglect

- Representational Neglect

\section{Relevant activities affected:}

- Communication

- Reading and writing

- Mobility and moving around

- Walking

- Dressing

- Toileting

- Washing oneself

- Transferring oneself

- Eating and drinking

- Telling the time

Relevant contextual factors affected: - Health professionals and caregivers

- Heatlh services

- Products for personal communication

- Relationships, family and support
Relevant restricions in participation: - preparation and consumption of meals - participation in traffic and public transport

- Recreation

- Work and labour

$\begin{array}{ll}\text { Diagnostics for neglect } & \\ \text { - Star cancellation test } & \text { - Comb and Razor test } \\ \text { - Line bisection test } & \text { - Eyetracking oculography } \\ \text { - Reading test } & \text { - Test for attentional performace } \\ \text { - Copying figures and drawings } & \text { - Virtual reality } \\ \text { - Behavioral inattention test } & \text { - Clinical observation } \\ \text { - Vienna test system } & \text { - Random shape cancellation test } \\ \text { - Two part picture test } & \text { - Munich reading test }\end{array}$

\section{Body function (impairments)}

\section{Neurological scales}

- Glasgow coma scale

- Mini mental state examination

- National institutes of Health stroke

scale

- Scandinavian stroke scale

- Canadian neurological scale

\section{Activity (disability)}

\section{ADL-scales}

- Catherine Bergego scale

- Barthel index

- Lucerne ICF-based Multidisciplinary

Observation Scale

- Functional independece measure

- Frenchay activities index

- (modified) Rankin scale

\section{Contextual factors}

- Caregiver strain index

- Family assessment device

\author{
Participation (handicap) \\ - Euroqol-5D \\ - Frenchay activities index \\ - Nottingham extended activities of daily \\ living \\ - Nottingham health profile \\ - General health questionnaire \\ - Stroke impact profile \\ - Medical outcome study short form 36 \\ - Stroke-specific quality of life
}

Fig. 1. The international classification of function, disability, and health framework for the effect of unilateral neglect on an individual. A. the ICF-model. B. most relevant categories affected by unilateral neglect. C. examples of commonly used measurement scales in the corresponding categories. Abbreviations: ADL = activities of daily living. Adapted from Langhorne et al. (2011), Plummer et al. (2003), and Ting et al. (2011). 


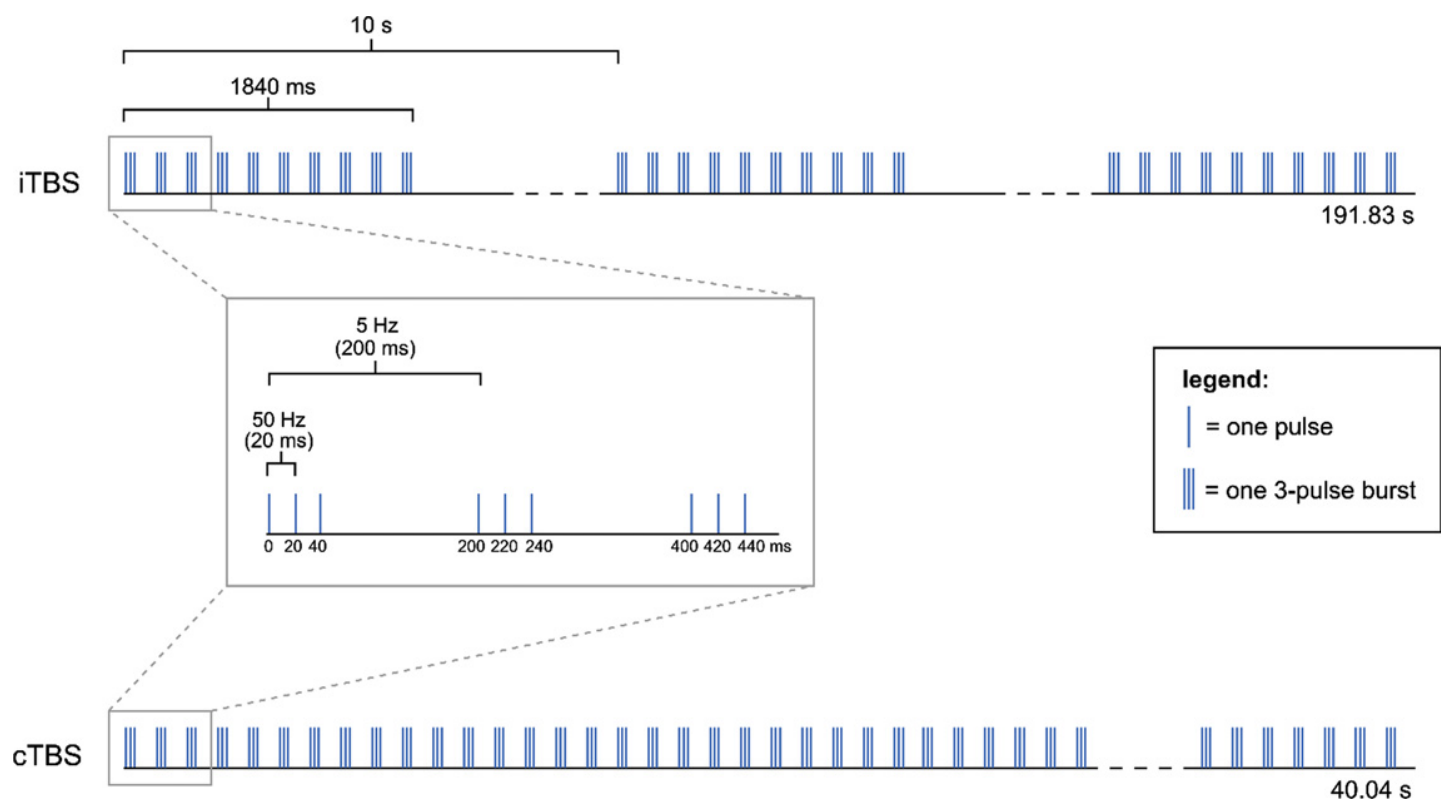

Fig. 2. Different TBS modalities used by Huang et al. (2005): iTBS and cTBS. The pattern consists of 3 pulses at $50 \mathrm{~Hz}$ repeated at $5 \mathrm{~Hz}$ (theta rhythm). In the iTBS protocol, groups of 10 bursts (i.e. trains) are repeated every $10 \mathrm{~s}$ for $191.84 \mathrm{~s}$, resulting in a total of 600 pulses in 20 trains. In the cTBS protocol, an uninterrupted train of 200 bursts is delivered for a total of $40.04 \mathrm{~s}$, resulting in a total of 600 pulses in 1 train.

formulated by Kinsbourne (Cazzoli et al., 2009; Kinsbourne, 1970, 1977), and functional-anatomical attention network models (Baldassarre et al., 2014; Koch et al., 2011). The rivalry model proposes that normal orienting of spatial attention involves a competition of the hemispheres to direct attention to a particular side in space. This competition is balanced by mutual interhemispheric transcallosal inhibition. A uni-hemispheric lesion, leading to a hypoactive ipsilesional hemisphere and a hyperactive contralesional hemisphere, is thought to induce an imbalance within this competitive attentional network. This imbalance biases spatial attention to the ipsilesional side of space, resulting in the symptoms of UN. Excitatory and inhibitory rTMS protocols can potentially be used to restore interhemispheric balance. There is indeed evidence that rTMS over the contralesional hemisphere can lead to a temporary reduction in errors in line bisection tasks (Oliveri et al., 2001), which underscores the notion that UN is characterized by an interhemispheric imbalance. Still, the response of UN to NIBS intervention is poorly understood, as a high variability in effectiveness of NIBS protocols has been reported (Lefaucheur et al., 2014). Interestingly, some studies suggest that the contralesional undamaged hemisphere should be facilitated rather than inhibited, pointing to a compensatory role of the undamaged hemisphere (Lunven et al., 2015; Umarova et al., 2016). This pattern of reorganization and compensation by the undamaged hemisphere is referred to as the vicariation model (Di Pino et al., 2014).

Theta-burst stimulation (TBS) is a patterned form of rTMS which can be delivered either continuously (cTBS) or intermittently (iTBS), and can thereby decrease or increase the excitability of corticospinal neurons under the stimulation site, respectively (Di Pino et al., 2014). The non-invasive stimulation of brain areas is performed using a magnetic field generated by an electrical current which passes through an inductive coil (Huang et al., 2005). TBS with humans was first introduced by Huang and colleagues in 2005, to stimulate the motor cortex (Huang et al., 2005), and was based on earlier results obtained from animal studies. Huang and colleagues (2005) proposed a protocol consisting of bursts of three pulses with $20 \mathrm{~ms}$ inter-pulse intervals $(50 \mathrm{~Hz})$, that repeats every $200 \mathrm{~ms}$ ( $5 \mathrm{~Hz}$; i.e. theta rhythm) (Fig. 2). The magnetic stimulation was given using a figure-ofeight coil, which was placed tangentially to the scalp. Even though variations of Huang's TBS protocol with modified stimulation parameters (e.g. $30 \mathrm{~Hz}$ bursts repeated at $5 \mathrm{~Hz}$ ) have been developed, the basic theta rhythm is maintained across these variations. 
Recent studies have looked specifically at TBS as a promising new protocol to target some of the symptoms of UN. It is thought that both modalities of TBS (i.e. iTBS and cTBS) can induce longterm changes in cortical excitability, being sustained beyond the time of stimulation (Huang et al., 2005). These long-lasting after-effects are especially present with repeated sessions of stimulation (Nyfeller et al., 2006), and can last longer than those known in other rTMS protocols (i.e. LF rTMS and HF rTMS) (Cárdenas-Morales et al., 2010), even though TBS employs a lower stimulation intensity and shorter stimulation time than conventional rTMS. Because of these advantages, TBS is proposed as a feasible, cost-effective and long-lasting therapeutic option for stroke patients with UN.

\subsection{Objectives of the present review}

The main objective of the present study is to systematically review the available literature (RCTs only), investigating whether TBS (including iTBS and cTBS) of the contralesional hemisphere is effective in improving symptoms of unilateral neglect in patients with right hemisphere stroke. The ICF-model will be used as a framework to categorize the effects of TBS on different outcome measures.

\section{Methods}

\subsection{Search strategy}

The methods of this systematic review were according to Preferred Reporting Items for Systematic Reviews and Meta-Analysis (PRISMA) (Moher et al., 2009). A comprehensive literature search was conducted for relevant RCTs using the following electronic databases: MEDLINE via PubMed, EMBASE via Ovid, and Cochrane Library. These databases were searched from inception up to the 27 th of February 2021. The search strategy was based on keywords relevant to the research question. These keywords were "transcranial magnetic stimulation", "stroke", and "unilateral neglect". For each individual database, a search string was composed based on these keywords. To make the search as comprehensive as possible, synonyms and alternative terms of these keywords were also used. The keywords were combined with AND/OR operators (e.g., "transcranial magnetic stimulation" OR "repetitive transcranial magnetic stimulation" OR "non-invasive brain stimulation" OR "theta-burst stimulation" AND "stroke" OR "cerebrovascular accident" OR "CVA" OR "cerebrovascular apoplexy" AND "unilateral neglect" OR "hemispatial neglect" OR "visuomotor neglect"). The full search strategies can be found as supplementary material. In addition to the systematic search of these databases, reference lists of relevant articles were searched for potentially eligible studies.

\subsection{Study selection}

Records were amassed using the Mendeley Desktop reference manager (version 1.19.8). After the duplicate records were removed, two assessors (M.H. and S.C.) independently screened the articles for eligibility, first based on title and abstract and thereafter based on full-text. A priori eligibility criteria were based on specific study components defined under the acronym PICOS (i.e. Population, Intervention, Comparison, Outcome, Study design) (Moher et al., 2009). The eligibility criteria, and the rationale behind the criteria, are provided in Table 1. Disagreements between the two assessors concerning study eligibility were resolved by discussion until consensus was reached. In the event agreement concerning the eligibility of a study could not be reached, a third independent assessor (J.S.) acted as adjudicator.

\subsection{Methodological assessment}

Included studies were assessed for methodological quality and risk of bias by two independent assessors (M.H. and S.C.) using the Physiotherapy Evidence Database (PEDro) scale, which is widely used to assess trials in physical therapy and rehabilitation (Moseley et al., 2002). This tool contains 11 items considering two aspects of methodological quality of RCTs, namely internal validity and whether the article presents sufficient statistical information to be interpretable. Each RCT was given a total PEDro score ranging from 0 to 10 , with higher scores indicating higher methodological quality (Moseley et al., 2002) (Table 3). The total score is based on items 2 to 11 . Disagreements between the two assessors concerning the PEDro score were resolved by discussion until consensus was reached or, if necessary, a third independent assessor (J.S.) decided on the final score. The PEDro scores were used as a basis for best-evidence synthesis and to discuss limitations and strengths of the included studies. PEDro scores were therefore not included in the eligibility criteria. 
Table 1

PICOS-table of eligibility criteria

\begin{tabular}{|c|c|}
\hline Component & Criteria \\
\hline Population & $\begin{array}{l}\text { - Human adults (over } 18 \text { years old), both males and females, with unilateral neglect after } \\
\text { right hemisphere stroke (ischemia or haemorrhage). } \\
\text { - Studies with stroke patients in all phases of stroke recovery (i.e. acute, subacute and } \\
\text { chronic) were included. } \\
\text { - No restrictions regarding diagnosis of neglect were applied, due to diagnostic difficulties } \\
\text { and heterogeneity of neglect symptoms. }\end{array}$ \\
\hline Intervention & $\begin{array}{l}\text { - Only studies with rTMS intervention with a TBS protocol were included; all other rTMS } \\
\text { intervention modalities, e.g. LF rTMS, were excluded. } \\
\text { - Studies with TBS intervention in combination with other forms of therapy (i.e. } \\
\text { conventional rehabilitation) were included. } \\
\text { - Stimulation is applied above the contralesional hemisphere. }\end{array}$ \\
\hline Comparison & - Comparison against sham stimulation and/or conventional rehabilitation. \\
\hline Outcome & $\begin{array}{l}\text { - Studies with outcome measures based on diagnostic instruments, measures of } \\
\text { impairment, disability or handicap (see Fig. 1), measures of depression or anxiety, or } \\
\text { measures of quality of life were included. } \\
\text { - Studies with outcome measures based only on fMRI (i.e. neuroimaging studies) were } \\
\text { excluded, since the objective of the present study is to review effects on clinical } \\
\text { symptoms of neglect. }\end{array}$ \\
\hline Study design & $\begin{array}{l}\text { - Only Randomized Controlled Trials (RCTs) and Cross-Over Randomized Controlled } \\
\text { trials (CORCTs) were included. }\end{array}$ \\
\hline $\begin{array}{l}\text { Selection criteria for full-text } \\
\text { screening }\end{array}$ & $\begin{array}{l}\text { - Peer-reviewed article published in English. } \\
\text { - Full-text article is available. }\end{array}$ \\
\hline
\end{tabular}

Abbreviations: fMRI = functional magnetic resonance imaging; LF rTMS = low-frequency repetitive transcranial magnetic stimulation; $\mathrm{RCTs}=$ randomized controlled trials; $\mathrm{rTMS}=$ repetitive transcranial magnetic stimulation; TBS = theta-burst stimulation.

\subsection{Data extraction}

Of the studies eligible for inclusion, data were structurally extracted and study characteristics were summarized in a table. The following variables were extracted: study characteristics (i.e. author(s), year of publication, country, number of participants, diagnostic methods used), participant characteristics (i.e. age, time since onset of stroke, type of stroke, severity of stroke, form of neglect, existing visual field deficits, stage of stroke recovery), treatment protocol characteristics (i.e. stimulation site, stimulation intensity, number of pulses per session, number of sessions), outcome measures, results, adverse effects and follow-up. The phases of stroke recovery, defined by the time post stroke onset, that were used were: hyper acute (0-24 hours), acute (1-7 days), early subacute ( 7 days -3 months), late subacute (3-6 months), and chronic (>6 months) (Bernhardt et al., 2017).

\subsection{Best evidence synthesis}

A best evidence synthesis (BES) of the available literature was conducted using the criteria set out by van Tulder et al. (1999) and van Peppen et al. (2004), which is based on the number of high-quality RCTs performed with statistically significant findings, within a certain category. Studies with a PEDro score of 4 or higher were deemed of high methodological quality (van Peppen et al., 2004). Effectiveness of the intervention was based on the reported statistical significance of the results at a threshold of $p<0.05$. Statistical significance was used, as a minimal clinically important difference has not been established for the outcome measures that assess neglect severity. The scale of the BES consists of five levels of evidence: (1) strong evidence; (2) moderate evidence; (3) limited evidence; (4) indicative findings; and (5) no or insufficient evidence (van Peppen et al., 2004). Table 2 provides information about when a certain level of evidence has been reached. Note that the presence of evidence does not necessarily mean that the intervention has favorable effects; evidence can be in favor of either the intervention or the control group.

The results of the systematic review were divided in different categories based on the following study characteristics: intervention protocol, location of stimulation, outcome measures, and time of measurement (i.e., distinction between short-term and long-term effects). The outcome measures used in the studies determined which health aspects classified by the ICF-model were being measured. Outcome measures based on diagnostic instruments were categorized as measures of neglect severity. 
Table 2

Best-evidence synthesis by van Tulder et al. (1999) and van Peppen et al. (2004)

\begin{tabular}{ll}
\hline Level of evidence & Criteria \\
\hline Strong evidence & $\begin{array}{c}\text { Provided by generally consistent statistically significant findings in outcome measures in at least two } \\
\text { high-quality RCTs, with PEDro scores of at least 4 points* } \\
\text { Provided by generally consistent statistically significant findings in outcome measures in at least one } \\
\text { high-quality RCT and at least one low-quality RCT ( } \leq 3 \text { points on PEDro) or one high-quality CCT* } \\
\text { Provided by generally consistent statistically significant findings in outcome measures in at least one } \\
\text { high-quality RCT or at least two high-quality CCTs* (in the absence of high-quality RCTs) } \\
\text { Provided by generally consistent statistically significant findings in outcome measures in at least one } \\
\text { high-quality CCT or low-quality RCTs* (in the absence of high-quality RCTs), or two studies of a } \\
\text { nonexperimental nature with sufficient quality (in the absence of RCTs and CCTs)* }\end{array}$ \\
$\begin{array}{c}\text { In the case that results of eligible studies do not meet the criteria for one of the above stated levels of evidence, or } \\
\text { in the case of conflicting (statistically significant positive and statistically significant negative) results among } \\
\text { RCTs and CCTs, or in the case of no eligible studies }\end{array}$ \\
\hline
\end{tabular}

Adapted from van Peppen et al. (2004). Abbreviations: CCT = controlled clinical trial; PEDro= Physiotherapy Evidence Database; $\mathrm{RCT}=$ randomized controlled trial. *If the number of studies that show evidence is $<50 \%$ of the total number of studies found within the same category of methodological quality and study design (e.g. RCTs in this review), no evidence will be classified.
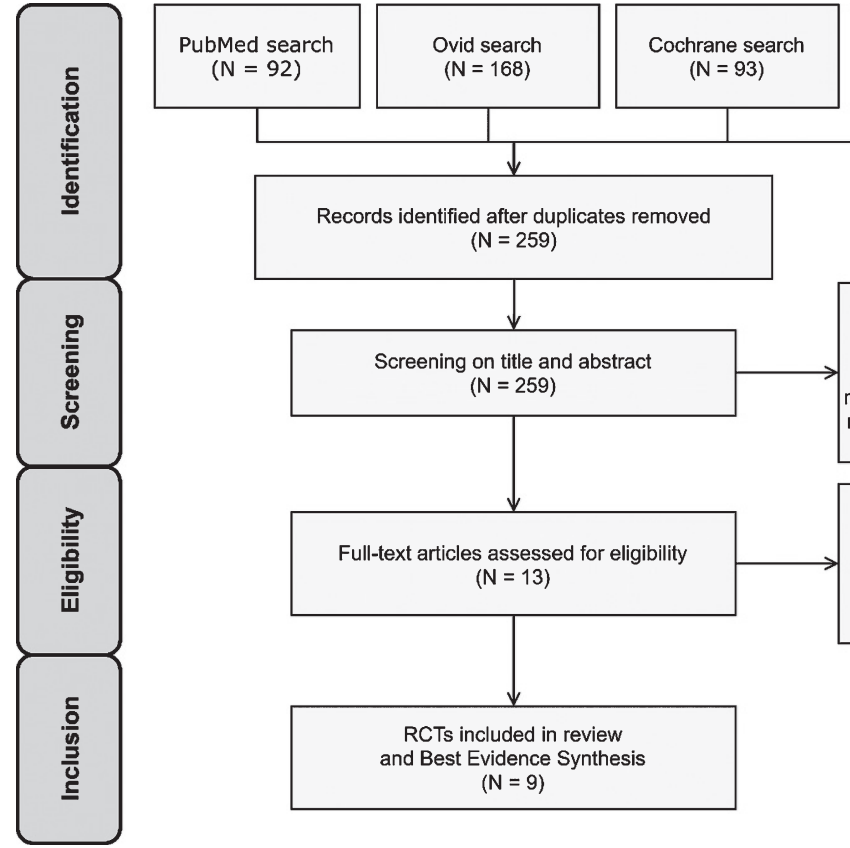

Fig. 3. PRISMA flow diagram for study selection.

\section{Results}

\subsection{Study characteristics}

A total of 353 potentially relevant publications was identified through the literature search and 259 remained after removing duplicates. 246 articles were excluded after title and abstract screening. 13 studies were thus assessed for eligibility by full-text screening. Nine studies met the eligibility criteria and were included in the review and BES (Cao et al., 2016;
Cazzoli et al., 2012; Fu et al., 2015, 2017; Hopfner et al., 2015; Koch et al., 2012; Nyffeler et al., 2019; Vatanparasti et al., 2019; Yang et al., 2015) (Fig. 3). All identified records originated from the systematic database search. No records from other sources (i.e. reference lists) were included, since all potentially eligible studies found in recent articles were already identified through the systematic database search.

The methodological quality scores of the included studies ranged from 5 to 9 on the PEDro scale (Table 3). This means that all included studies are 
Table 3

Methodological quality of the included studies based on the PEDro scale

\begin{tabular}{|c|c|c|c|c|c|c|c|c|c|c|c|c|}
\hline \multirow[b]{2}{*}{ Studies } & \multicolumn{12}{|c|}{ PEDro items } \\
\hline & 1 & 2 & 3 & 4 & 5 & 6 & 7 & 8 & 9 & 10 & 11 & Score \\
\hline Cao et al., 2016 & $\mathrm{Y}$ & 1 & 0 & 1 & 0 & 0 & 0 & 0 & 1 & 1 & 1 & 5 \\
\hline Fu et al., 2015 & $\mathrm{Y}$ & 1 & 0 & 1 & 1 & 1 & 1 & 1 & 1 & 1 & 1 & 9 \\
\hline Fu et al., 2017 & $\mathrm{Y}$ & 1 & 0 & 1 & 1 & 0 & 0 & 0 & 1 & 1 & 1 & 6 \\
\hline Hopfner et al., 2015 & $\mathrm{~N}$ & 1 & 0 & 1 & 0 & 0 & 0 & 0 & 1 & 1 & 1 & 5 \\
\hline Nyffeler et al., 2019 & $\mathrm{Y}$ & 1 & 1 & 1 & 1 & 0 & 1 & 1 & 1 & 1 & 1 & 9 \\
\hline Vatanparasti et al., 2019 & $\mathrm{Y}$ & 1 & 0 & 1 & 1 & 0 & 0 & 1 & 1 & 1 & 1 & 7 \\
\hline Yang et al., 2015 & $\mathrm{Y}$ & 1 & 0 & 1 & 0 & 0 & 0 & 1 & 1 & 1 & 1 & 6 \\
\hline Total score per item & & 9 & 1 & 9 & 6 & 2 & 3 & 6 & 9 & 9 & 9 & \\
\hline
\end{tabular}

Adapted from Moseley et al. (2002). Note: 1: Eligibility criteria were specified; 2: Subjects were randomly allocated to groups (in a crossover study, subjects were randomly allocated an order in which treatments were received); 3: Allocation was concealed; 4: The groups were similar at baseline regarding the most important prognostic indicators; 5: There was blinding of all subjects; 6 : There was blinding of all therapists who administered the therapy; 7: There was blinding of all assessors who measured at least one key outcome; 8: Measures of at least one key outcome were obtained from $>85 \%$ of the subjects initially allocated to groups; 9 : All subjects for whom outcome measures were available received the treatment or control condition as allocated or, where this was not the case, data for at least one key outcome were analyzed by intention to treat; 10: The results of between-group statistical comparisons are reported for at least one key outcome; 11: The study provides both point measures and measures of variability for at least one key outcome. All items except item 1 were scored on a scale of $0-10$; this resulted in a total PEDro score for each RCT ranging from 0 to 10 , with higher scores indicating higher methodological quality.

deemed of high quality. Clinical and demographical features of the included studies are provided in Table 4. Altogether, a total of 187 participants were included in these studies. Nyfeller et al. (2019) tested an additional 30 stroke patients without $\mathrm{UN}$ as extra control, these are not included in the total participant count. Individual sample sizes ranged from 12 to 38 participants. Participants had a mean age of 60.27 years. Most participants, of those reported, suffered an ischemic stroke ( $N=68$ ischemic; $N=46$ haemorrhagic; $N=73$ not reported) and most were male ( $N=108$ male, $N=61$ female; $N=18$ not reported). Lesion volumes, or a different measure of stroke severity, were reported by only two out of the nine included studies (Cazzoli et al., 2012; Nyffeler et al., 2019). The stages of stroke recovery where participants were in, as determined by the mean time since onset of stroke, were early or late subacute (Cao et al., 2016; Cazzoli et al., 2012; Fu et al., 2015, 2017; Hopfner et al., 2015; Koch et al., 2012; Nyffeler et al., 2019; Vatanparasti et al., 2019; Yang et al., 2015), and chronic (Vatanparasti et al., 2019). Terms used to classify the type of neglect varied among studies. The terms used were spatial neglect (Cazzoli et al., 2012; Hopfner et al., 2015; Nyffeler et al., 2019), unilateral spatial neglect (Yang et al., 2015), visual spatial neglect (Cao et al., 2016), visuospatial neglect (Fu et al., 2015, 2017), visuospatial unilateral neglect (Vatanparasti et al., 2019), and hemispatial neglect (Koch et al., 2012).

\subsection{Treatment protocols and concurrent therapy}

In eight out of nine of the included studies, cTBS was delivered over the left contralesional posterior parietal cortex (PPC) (Cazzoli et al., 2012; Fu et al., 2015, 2017; Hopfner et al., 2015; Koch et al., 2012; Nyffeler et al., 2019; Vatanparasti et al., 2019; Yang et al., 2015). One study delivered iTBS over the left contralesional dorsolateral prefrontal cortex (DLPFC) (Cao et al., 2016). The TBS protocols and number of sessions varied across studies (Table 5). Variability in TBS protocols originated mainly in the inter-burst frequencies and in the total number of pulses delivered. Inter-pulse frequencies were $50 \mathrm{~Hz}$ in two studies (Cao et al., 2016; Koch et al., 2012), and $30 \mathrm{~Hz}$ in the other seven studies (Cazzoli et al., 2012; Fu et al., 2015, 2017; Hopfner et al., 2015; Nyffeler et al., 2019; Vatanparasti et al., 2019; Yang et al., 2015). The number of sessions per day ranged from 1 to 10 , and treatment duration ranged from 1 day to 14 days.

Many different forms of concurrent therapy, which participants received regardless of group allocation, were used in the included studies: visuospatial scanning training, movement function training, attention and concentration training, occupational therapy, physiotherapy, smooth pursuit eye movement training, motor rehab, speech therapy, and interdisciplinary therapy (Table 4). 
Table 4

Clinical and demographical features of the included studies

\begin{tabular}{|c|c|c|c|c|c|c|c|c|c|}
\hline $\begin{array}{l}\text { Author(s); } \\
\text { Publication year; } \\
\text { Location } \\
\end{array}$ & $\begin{array}{l}\text { No. of } \\
\text { participants }\end{array}$ & $\begin{array}{l}\text { Sex (Male/ } \\
\text { Female) }\end{array}$ & Age (years) & $\begin{array}{l}\text { Type of stroke } \\
\text { (IS/HAE) }\end{array}$ & Lesion volume $\left(\mathrm{cm}^{3}\right)$ & $\begin{array}{l}\text { Time since onset of } \\
\text { stroke }\end{array}$ & $\begin{array}{l}\text { Form of neglect; } \\
\text { Diagnostic methods }\end{array}$ & $\begin{array}{l}\text { Visual acuity; central } 30^{\circ} \\
\text { of visual field }\end{array}$ & Concurrent therapy \\
\hline $\begin{array}{l}\text { Cao et al., } 2016 \\
\text { China }\end{array}$ & $\begin{array}{l}\text { Tot: } 13 \\
\text { I: } 7 \\
\text { C: } 6 \\
\end{array}$ & $\begin{array}{l}\text { Tot: } 11 / 2 \\
\text { I: } 6 / 1 \\
\text { C: } 5 / 1 \\
\end{array}$ & $\begin{array}{l}(\text { mean } \pm \text { SD }) \\
\text { I: } 55.0 \pm 12.0 \\
\text { C: } 62.0 \pm 10.0\end{array}$ & N/A & N/A & $\begin{array}{l}\text { (mean } \pm \text { SD) } \\
\text { I: } 32.0 \pm 17.0 \text { days } \\
\text { C: } 36.0 \pm 17.0 \text { days }\end{array}$ & $\begin{array}{l}\text { Visual spatial neglect; } \\
\text { LBT }\end{array}$ & $\begin{array}{l}\text { Normal or corrected; } \\
\text { intact }\end{array}$ & VST, MFT \\
\hline $\begin{array}{l}\text { Cazzoli et al., } \\
2012 \\
\text { Switzerland }\end{array}$ & $\begin{array}{l}\text { Tot: } 24 \\
\mathrm{I}_{1}: 8 \\
\mathrm{I}_{2}: 8 \\
\mathrm{C}: 8 \\
\end{array}$ & Tot: $17 / 7$ & $\begin{array}{l}\text { (mean } \pm \text { SEM }) \\
\text { Tot: } 58 \pm 2.25\end{array}$ & Tot: $14 / 10$ & $\begin{array}{l}\text { mean } \pm \text { SEM }) \\
\mathrm{I}_{1}: 61.9 \pm 9.35 \\
\mathrm{I}_{2}: 122.72 \pm 31.14 \\
\text { C: } 57.99 \pm 20.19 \\
\end{array}$ & $\begin{array}{l}\text { (mean } \pm \text { SEM) } \\
\text { Tot: } 26.63 \pm 4.44 \text { days }\end{array}$ & $\begin{array}{l}\text { Spatial neglect; } \\
\text { cancellation task, } \\
\text { drawing task, LBT }\end{array}$ & $\begin{array}{l}\text { Normal or corrected; } \\
\text { intact }\end{array}$ & VST, A\&CT, OT, PT \\
\hline $\begin{array}{l}\text { Fu et al., } 2015 \\
\text { China }\end{array}$ & $\begin{array}{l}\text { Tot: } 20 \\
\text { I: } 10 \\
\text { C: } 10\end{array}$ & $\begin{array}{l}\text { Tot: } 16 / 4 \\
\text { I: } 8 / 2 \\
\text { C: } 8 / 2\end{array}$ & $\begin{array}{l}(\text { Mean } \pm \text { SD }) \\
\text { Tot: } 57.3 \pm 13.2 \\
\text { I: } 55.1 \pm 14.0 \\
\text { C: } 59.5 \pm 12.7 \\
\end{array}$ & $\begin{array}{l}\text { Tot: } 9 / 11 \\
\text { I: } 5 / 5 \\
\text { C: } 6 / 4\end{array}$ & N/A & $\begin{array}{l}(\text { mean } \pm \text { SD }) \\
\text { Tot: } 42.6 \pm 26.3 \text { days } \\
\text { I: } 50.3 \pm 33.3 \text { days } \\
\text { C: } 34.9 \pm 14.6 \text { days }\end{array}$ & $\begin{array}{l}\text { Visuospatial neglect; } \\
\text { SCT, LBT }\end{array}$ & $\begin{array}{l}\text { Normal or corrected; } \\
\text { patients with VD } \\
\text { excluded }\end{array}$ & VST \\
\hline $\begin{array}{l}\text { Fu et al., } 2017 \\
\text { China }\end{array}$ & $\begin{array}{l}\text { Tot: } 12 \\
\text { I: } 6 \\
\text { C: } 6 \\
\end{array}$ & Tot: $9 / 3$ & $\begin{array}{l}(\text { mean } \pm \text { SD) } \\
\text { I: } 60.2 \pm 14.1 \\
\text { C: } 62.0 \pm 9.8 \\
\end{array}$ & N/A & N/A & $\begin{array}{l}(\text { mean } \pm \text { SD) } \\
\text { I: } 41.8 \pm 20.6 \text { days } \\
\text { C: } 36.2 \pm 17.5 \text { days }\end{array}$ & $\begin{array}{l}\text { Visuospatial neglect; } \\
\text { SCT, LBT }\end{array}$ & $\begin{array}{l}\text { Normal or corrected; } \\
\text { patients with VD } \\
\text { excluded }\end{array}$ & VST, MFT \\
\hline $\begin{array}{l}\text { Hopfner et al., } \\
2015 \\
\text { Switzerland } \\
\end{array}$ & $\begin{array}{l}\text { Tot: } 18 \\
\mathrm{I}_{1+2}: 12 \\
\mathrm{C}_{1+2}: 6 \\
\end{array}$ & Tot: $9 / 9$ & $\begin{array}{l}\text { (mean } \pm \text { SD) } \\
\text { Tot: } 64.5 \pm 12.1\end{array}$ & Tot: $13 / 5$ & $\mathrm{~N} / \mathrm{A}$ & $\begin{array}{l}\text { (mean } \pm \text { SD) } \\
\text { Tot: } 31.9 \pm 14.1 \text { days }\end{array}$ & $\begin{array}{l}\text { Spatial neglect; } \\
\text { LBT, BT }\end{array}$ & $\begin{array}{l}\text { Normal or corrected; } \\
\text { intact }\end{array}$ & SPT \\
\hline $\begin{array}{l}\text { Koch et al., } 2012 \\
\text { Italy }\end{array}$ & $\begin{array}{l}\text { Tot: } 18 \\
\text { I: } 9 \\
\text { C: } 9 \\
\end{array}$ & N/A & $\begin{array}{l}\text { (mean } \pm \text { SEM) } \\
\text { Tot: } 66.7 \pm 2.6\end{array}$ & N/A & N/A & $\begin{array}{l}\text { (mean } \pm \text { SEM) } \\
\text { Tot: } 43.4 \pm 5.3 \text { days }\end{array}$ & $\begin{array}{l}\text { Hemispatial neglect; } \\
\text { Clinical examination, } \\
\text { BIT }\end{array}$ & N/A & VST, A\&CT, motor rehab \\
\hline $\begin{array}{l}\text { Nyffeler et al., } \\
2019 \\
\text { Switzerland }\end{array}$ & $\begin{array}{l}\text { Tot: } 30 \\
\\
\mathrm{I}_{1}: 10 \\
\mathrm{I}_{2}: 10 \\
\mathrm{C}: 10 \\
\end{array}$ & $\begin{array}{l}\text { Tot: } 18 / 12 \\
\mathrm{I}_{1}: 5 / 5 \\
\mathrm{I}_{2}: 6 / 4 \\
\mathrm{C}: 7 / 3 \\
\end{array}$ & $\begin{array}{l}\text { (mean } \pm \text { SD) } \\
\\
\mathrm{I}_{1}: 67.80 \pm 10.13 \\
\mathrm{I}_{2}: 74.30 \pm 20.23 \\
\text { C: } 70.60 \pm 11.44 \\
\end{array}$ & N/A & $\begin{array}{l}\text { (mean } \pm \mathrm{SD}) \\
\\
\mathrm{I}_{1}: 79.101 \pm 62.39 \\
\mathrm{I}_{2}: 99.92 \pm 83.55 \\
\mathrm{C}: 105.30 \pm 112.24 \\
\end{array}$ & $\begin{array}{l}\text { (mean } \pm \mathrm{SD}) \\
\\
\mathrm{I}_{1}: 26.8 \pm 20.89 \text { days } \\
\mathrm{I}_{2}: 22.90 \pm 10.34 \text { days } \\
\text { C: } 25.8 \pm 11.26 \text { days }\end{array}$ & $\begin{array}{l}\text { Spatial neglect; } \\
\text { CBS, LBT, BT }\end{array}$ & N/A & $\begin{array}{l}\text { SPT, interdisciplinary } \\
\text { therapy }\end{array}$ \\
\hline $\begin{array}{l}\text { Vatanparasti } \\
\text { et al., } 2019 \text { Iran }\end{array}$ & $\begin{array}{l}\text { Tot: } 14 \\
\text { I: } 7 \\
\text { C: } 7 \\
\end{array}$ & $\begin{array}{l}\text { Tot: } 10 / 4 \\
\text { I: } 5 / 2 \\
\text { C } 5 / 2 \\
\end{array}$ & $\begin{array}{l}(\mathrm{mean} \pm \mathrm{SD}) \\
\text { I: } 67.5 \pm 8.4 \\
\text { C: } 65.5 \pm 10.2\end{array}$ & $\begin{array}{l}\text { Tot: } 8 / 6 \\
\text { I: } 4 / 3 \\
\text { C: } 4 / 3 \\
\end{array}$ & N/A & $\begin{array}{l}\text { (<6 months / chronic) } \\
\text { I: } 3 / 4 \\
\text { C: } 3 / 4\end{array}$ & $\begin{array}{l}\text { Visuospatial unilateral } \\
\text { neglect } \\
\text { clinical examination }\end{array}$ & N/A & PA \\
\hline $\begin{array}{l}\text { Yang et al., } 2015 \\
\text { China }\end{array}$ & $\begin{array}{l}\text { Tot: } 38 \\
\mathrm{I}_{1}: 9 \\
\mathrm{I}_{2}: 10 \\
\mathrm{I}_{3}: 9 \\
\mathrm{C}: 10 \\
\end{array}$ & $\begin{array}{l}\text { Tot: } 18 / 20 \\
\mathrm{I}_{1}: 6 / 3 \\
\mathrm{I}_{2}: 4 / 6 \\
\mathrm{I}_{3}: 5 / 4 \\
\mathrm{C}: 3 / 7 \\
\end{array}$ & $\begin{array}{l}(\operatorname{mean} \pm \mathrm{SD}) \\
\mathrm{I}_{1}: 46.72 \pm 13.11 \\
\mathrm{I}_{2}: 48.01 \pm 12.25 \\
\mathrm{I}_{3}: 49.45 \pm 10.78 \\
\text { C: } 47.70 \pm 11.81 \\
\end{array}$ & $\begin{array}{l}\text { Tot: } 24 / 14 \\
\mathrm{I}_{1}: 5 / 4 \\
\mathrm{I}_{2}: 7 / 3 \\
\mathrm{I}_{3}: 6 / 3 \\
\mathrm{C}: 6 / 4 \\
\end{array}$ & $\mathrm{~N} / \mathrm{A}$ & $\begin{array}{l}\text { (mean } \pm \text { SD) } \\
\mathrm{I}_{1}: 100.96 \pm 38.52 \text { days } \\
\mathrm{I}_{2}: 107.52 \pm 39.24 \text { days } \\
\mathrm{I}_{3}: 104.85 \pm 36.38 \text { days } \\
\mathrm{C}: 105.91 \pm 37.59 \text { days }\end{array}$ & $\begin{array}{l}\text { Unilateral spatial neglect; } \\
\text { SCT, LBT, clinical } \\
\text { examination }\end{array}$ & $\begin{array}{l}\text { N/A; } \\
\text { Patients with VD } \\
\text { excluded }\end{array}$ & OT, PT, speech training \\
\hline
\end{tabular}

MFT = movement function training; N/A=not available; OT = occupational therapy; $\mathrm{PA}=$ Prism Adaptation; $\mathrm{PT}=$ physiotherapy; $\mathrm{RLCT}=$ random letter cancellation test $\mathrm{SCT}=$ star cancellation test $\mathrm{SD}=$ standard deviation; $\mathrm{SEM}=$ Standard Error of the Mean; $\mathrm{SPT}=$ smooth pursuit eye movement training; Tot = total sample; VD= visual deficits; VST = visuospatial scanning training. 
Table 5

Intervention protocols and main results of the included studies

\begin{tabular}{|c|c|c|c|c|c|c|c|c|c|}
\hline \multirow[t]{2}{*}{$\overline{\text { Study }}$} & \multirow[t]{2}{*}{ Design; groups } & \multirow[t]{2}{*}{ Stim. site } & \multirow[t]{2}{*}{$\begin{array}{l}\text { TBS Intervention } \\
\text { protocol }\end{array}$} & \multirow[t]{2}{*}{ Comparison } & \multirow[t]{2}{*}{ No. of sessions } & \multirow[t]{2}{*}{$\begin{array}{l}\text { Between-group } \\
\text { outcome measures }\end{array}$} & \multicolumn{2}{|c|}{$\begin{array}{l}\text { Between-group } \\
\text { results }\end{array}$} & \multirow[t]{2}{*}{ Follow-up time } \\
\hline & & & & & & & Immediate & Follow-up & \\
\hline $\begin{array}{l}\text { Cao et al., } 2016 \\
\text { China }\end{array}$ & $\begin{array}{l}\text { RCT; } \\
\text { I= iTBS and CT } \\
\text { C= low-intensity } \\
\quad \text { iTBS and CT }\end{array}$ & $\begin{array}{l}\text { Left contra- } \\
\text { lesional } \\
\text { DLPFC } \\
\text { (F5) }\end{array}$ & $\begin{array}{l}1 \text { session }=20 \text { trains of } \\
\text { 3-pulse } 50 \mathrm{~Hz} \text { bursts, } \\
\text { repeated at } 5 \mathrm{~Hz} \text {, with } \\
\text { an inter-train interval } \\
\text { of } 8 \mathrm{sec} \text {, at } 80 \% \text { RMT }\end{array}$ & $\begin{array}{l}1 \text { session }=20 \text { trains of } \\
\text { 3-pulse } 50 \mathrm{~Hz} \text { bursts, } \\
\text { repeated at } 5 \mathrm{~Hz} \text {, with } \\
\text { an inter-train interval } \\
\text { of } 8 \mathrm{sec} \text {, at } 40 \% \text { RMT }\end{array}$ & $\begin{array}{l}2 \text { sessions / day for } \\
10 \text { days }\end{array}$ & $\begin{array}{l}\text { I vs. C: } \\
\text { SCT } \\
\text { LBT }\end{array}$ & $\begin{array}{l}+ \\
+\end{array}$ & N/A & N/A \\
\hline $\begin{array}{l}\text { Cazzoli et al., } 2012 \\
\text { Switzerland }\end{array}$ & $\begin{array}{l}\text { CORCT; } \\
\mathrm{I}_{1}=\text { cTBS then } \\
\text { sham and CT } \\
\mathrm{I}_{2}=\text { sham then } \\
\text { cTBS and CT } \\
\mathrm{C}=\text { only CT }\end{array}$ & $\begin{array}{l}\text { Left contra- } \\
\text { lesional } \\
\text { PPC (P3) }\end{array}$ & $\begin{array}{l}1 \text { session = a } 44 \mathrm{~s} \\
\text { un-interrupted train } \\
\text { of 3-pulse } 30 \mathrm{~Hz} \\
\text { bursts, repeated at } \\
6 \mathrm{~Hz} \text {, at } 100 \% \mathrm{RMT} \text {; } \\
801 \text { total pulses }\end{array}$ & $\begin{array}{l}\text { Sham cTBS (coil } \\
\text { perpendicular to the } \\
\text { patient's scalp) and } \\
\text { no cTBS }\end{array}$ & $\begin{array}{l}4 \text { sessions/ day for } \\
2 \text { days }\end{array}$ & $\begin{array}{l}\text { cTBS }\left(\mathrm{I}_{1} \text { and } \mathrm{I}_{2}\right) \\
\text { vs. C: } \\
\text { CBS } \\
\text { VTS } \\
\text { RSCT } \\
\text { TPPT } \\
\text { MRT } \\
\text { cTBS vs. sham } \\
\text { (within } \mathrm{I}_{1} \text { and } \mathrm{I}_{2} \text { ): } \\
\text { CBS } \\
\text { VTS } \\
\text { RSCT } \\
\text { TPPT } \\
\text { MRT } \\
\end{array}$ & $\begin{array}{c}+ \\
+ \\
+ \\
+ \\
\text { NS } \\
\\
+ \\
+ \\
+ \\
+ \\
\text { NS }\end{array}$ & $\begin{array}{c}+ \\
+ \\
\text { NS } \\
\text { NS } \\
\text { NS }\end{array}$ & $\begin{array}{c}2 \text { weeks after las } \\
\text { cTBS session }\end{array}$ \\
\hline $\begin{array}{l}\text { Fu et al., } 2015 \\
\text { China }\end{array}$ & $\begin{array}{l}\text { RCT; } \\
\mathrm{I}=\mathrm{cTBS} \text { and CT } \\
\mathrm{C}=\text { sham and } \mathrm{CT} \text {. }\end{array}$ & $\begin{array}{l}\text { Left contra- } \\
\text { lesional } \\
\text { PPC (P5) }\end{array}$ & $\begin{array}{l}1 \text { session }=\text { a } 40 \mathrm{~s} \\
\text { un-interrupted train } \\
\text { of 3-pulse } 30 \mathrm{~Hz} \\
\text { bursts, repeated at } \\
5 \mathrm{~Hz} \text {, at } 80 \% \text { RMT; } \\
600 \text { total pulses }\end{array}$ & $\begin{array}{l}\text { Sham cTBS (coil } \\
\text { perpendicular to the } \\
\text { patient's scalp) }\end{array}$ & $\begin{array}{l}4 \text { sessions / day for } \\
14 \text { days }\end{array}$ & $\begin{array}{l}\text { I vs. C: } \\
\text { SCT } \\
\text { LBT }\end{array}$ & $\begin{array}{c}+ \\
\text { NS }\end{array}$ & $\begin{array}{l}+ \\
+\end{array}$ & $\begin{array}{c}4 \text { weeks after las } \\
\text { cTBS session }\end{array}$ \\
\hline $\begin{array}{l}\text { Fu et al., } 2017 \\
\text { China }\end{array}$ & $\begin{array}{l}\text { RCT; } \\
\mathrm{I}=\mathrm{cTBS} \text { and } \mathrm{CT} \\
\mathrm{C}=\text { low-intensity } \\
\text { cTBS and CT }\end{array}$ & $\begin{array}{l}\text { Left contra- } \\
\text { lesional } \\
\text { PPC (P3) }\end{array}$ & $\begin{array}{l}1 \text { session }=\text { a } 40 \mathrm{~s} \\
\text { un-interrupted train } \\
\text { of } 3 \text {-pulse } 30 \mathrm{~Hz} \\
\text { bursts, repeated at } \\
5 \mathrm{~Hz} \text {, at } 80 \% \text { RMT; } \\
600 \text { total pulses }\end{array}$ & $\begin{array}{l}1 \text { session }=\text { a } 40 \mathrm{sec} \\
\text { un-interrupted train } \\
\text { of } 3 \text {-pulse } 30 \mathrm{~Hz} \\
\text { bursts, repeated at } \\
5 \mathrm{~Hz} \text {, at } 40 \% \mathrm{RMT} \\
600 \text { total pulses }\end{array}$ & $\begin{array}{l}4 \text { sessions/ day for } \\
10 \text { days }\end{array}$ & $\begin{array}{l}\text { I vs. C: } \\
\text { SCT } \\
\text { LBT }\end{array}$ & $\begin{array}{l}+ \\
+\end{array}$ & N/A & N/A \\
\hline $\begin{array}{l}\text { Hopfner et al. } 2015 \\
\text { Switzerland }\end{array}$ & $\begin{array}{l}\text { CORCT; } \\
\mathrm{I}_{1}=\text { cTBS and CT } \\
\mathrm{I}_{2}=\text { sham and CT } \\
\mathrm{C}_{1}=\text { only cTBS } \\
\mathrm{C}_{2}=\text { only sham }\end{array}$ & $\begin{array}{l}\text { Left contra- } \\
\text { lesional } \\
\text { PPC (P3) }\end{array}$ & $\begin{array}{l}1 \text { session = a } 44 \mathrm{~s} \\
\text { un-interrupted train } \\
\text { of 3-pulse } 30 \mathrm{~Hz} \\
\text { bursts, repeated at } \\
6 \mathrm{~Hz} \text {, at } 100 \% \mathrm{RMT} \text {; } \\
801 \text { total pulses }\end{array}$ & $\begin{array}{l}\text { Sham cTBS (coil } \\
\text { perpendicular to the } \\
\text { patient's scalp) }\end{array}$ & 1 session on 1 day & $\begin{array}{l}\mathrm{I}_{1} \text { vs. } \mathrm{I}_{2}: \\
\text { BCT } \\
\mathrm{C}_{1} \text { vs. } \mathrm{C}_{2}: \\
\text { BCT } \\
\mathrm{I}_{1} \text { vs. } \mathrm{C}_{1}: \\
\text { BCT }\end{array}$ & $\begin{array}{l}+ \\
+ \\
\text { NS }\end{array}$ & N/A & N/A \\
\hline
\end{tabular}




\begin{tabular}{|c|c|c|c|c|c|c|c|c|c|}
\hline $\begin{array}{l}\text { Koch et al., } 2012 \\
\text { Italy }\end{array}$ & $\begin{array}{l}\text { RCT; } \\
\mathrm{I}=\mathrm{cTBS} \text { and } \mathrm{CT} \\
\mathrm{C}=\text { sham and } \mathrm{CT}\end{array}$ & $\begin{array}{l}\text { Left contra- } \\
\text { lesional } \\
\text { PPC }\end{array}$ & $\begin{array}{l}1 \text { session }=\text { a } 40 \mathrm{~s} \\
\text { un-interrupted train } \\
\text { of } 3 \text {-pulse } 50 \mathrm{~Hz} \\
\text { bursts, repeated at } \\
5 \mathrm{~Hz} \text {, at } 80 \% \text { AMT; } \\
600 \text { total pulses }\end{array}$ & $\begin{array}{l}\text { Sham cTBS (coil } \\
\text { perpendicular to the } \\
\text { patient's scalp) }\end{array}$ & $\begin{array}{l}2 \text { sessions / day for } \\
10 \text { days }\end{array}$ & $\begin{array}{l}\text { I vs. C: } \\
\text { BIT }\end{array}$ & + & + & $\begin{array}{l}2 \text { weeks after last } \\
\text { cTBS session }\end{array}$ \\
\hline $\begin{array}{l}\text { Nyffeler et al., } \\
2019 \text { Switzerland }\end{array}$ & $\begin{array}{l}\text { RCT; } \\
\mathrm{I}_{1}=8 \times \mathrm{cTBS} \text { and } \\
\text { CT } \\
\mathrm{I}_{2}=16 \times \mathrm{cTBS} \text { and } \\
\text { CT } \\
\mathrm{C}=\text { sham and } \mathrm{CT}\end{array}$ & $\begin{array}{l}\text { Left contra- } \\
\text { lesional } \\
\text { PPC (P3) }\end{array}$ & $\begin{array}{l}1 \text { session = a } 44 \mathrm{~s} \\
\text { un-interrupted train } \\
\text { of 3-pulse } 30 \mathrm{~Hz} \\
\text { bursts, repeated at } \\
6 \mathrm{~Hz} \text {, at } 100 \% \text { RMT; } \\
801 \text { total pulses }\end{array}$ & Sham cTBS (sham coil) & $\begin{array}{l}4 \text { sessions / day for } \\
\text { either } 2 \text { days }\left(I_{1}\right) \\
\text { or } 4 \text { days }\left(I_{2}\right)\end{array}$ & $\begin{array}{l}\text { I }_{1} \text { vs. C: } \\
\text { CBS } \\
\text { Test battery } \\
\text { FIM } \\
\text { LIMOS } \\
\text { I }_{2} \text { vs. C: } \\
\text { CBS } \\
\text { Test battery } \\
\text { FIM } \\
\text { LIMOS }\end{array}$ & $\begin{array}{l}+ \\
+ \\
+ \\
+ \\
+ \\
+ \\
+ \\
+ \\
+\end{array}$ & $\begin{array}{c}+ \\
+ \\
\text { N/A } \\
\text { N/A } \\
\\
+ \\
+ \\
\text { N/A } \\
\text { N/A }\end{array}$ & $\begin{array}{l}3 \text { months after last } \\
\text { cTBS session }\end{array}$ \\
\hline $\begin{array}{l}\text { Vatanparasti et al., } \\
2019 \text { Iran }\end{array}$ & $\begin{array}{l}\text { RCT; } \\
\mathrm{I}=\text { cTBS and CT } \\
\mathrm{C}=\text { sham and } \mathrm{CT}\end{array}$ & $\begin{array}{l}\text { Left contra- } \\
\text { lesional PPC } \\
\text { (P3) }\end{array}$ & $\begin{array}{l}1 \text { session }=\text { a } 26.77 \mathrm{~s} \text { un- } \\
\text { interrupted train of } \\
\text { 3-pulse } 30 \mathrm{~Hz} \text { bursts, } \\
\text { repeated at } 10 \mathrm{~Hz} \text {, at } \\
80 \% \text { RMT; } 801 \text { total } \\
\text { pulses }\end{array}$ & $\begin{array}{l}\text { Sham cTBS (coil } \\
\text { perpendicular to the } \\
\text { patient's scalp) }\end{array}$ & $\begin{array}{l}10 \text { sessions / day } \\
\text { for } 14 \text { days }\end{array}$ & $\begin{array}{l}\text { I vs. C: } \\
\text { SCT } \\
\text { LBT } \\
\text { Figure copying test } \\
\text { Clock drawing task } \\
\text { MRS }\end{array}$ & $\begin{array}{l}\text { NS } \\
\text { NS } \\
\text { NS } \\
\text { NS } \\
\text { NS }\end{array}$ & N/A & N/A \\
\hline $\begin{array}{l}\text { Yang et al., } 2015 \\
\text { China }\end{array}$ & $\begin{array}{l}\text { RCT; } \\
\mathrm{I}_{1}=1 \mathrm{~Hz} \text { rTMS } \\
\mathrm{I}_{2}=10 \mathrm{~Hz} \text { rTMS } \\
\mathrm{I}_{3}=\text { cTBS } \\
\mathrm{C}=\text { sham }\end{array}$ & $\begin{array}{l}\text { Left contra- } \\
\text { lesional } \\
\text { PPC (P3) }\end{array}$ & $\begin{array}{l}1 \text { session }=\text { an } \\
\text { uninter-rupted train } \\
\text { of } 3 \text {-pulse } 30 \mathrm{~Hz} \\
\text { bursts, repeated at } \\
5 \mathrm{~Hz} \text {, at } 80 \% \text { RMT; } \\
801 \text { total pulses }\end{array}$ & $\begin{array}{l}\text { Sham cTBS (coil } \\
\text { turned backwards), } \\
1 \text { Hz rTMS (i.e. LF } \\
\text { rTMS), } 10 \text { Hz rTMS } \\
\text { (i.e. HF rTMS) }\end{array}$ & $\begin{array}{l}2 \text { sessions / day for } \\
14 \text { days }\end{array}$ & $\begin{array}{l}\mathrm{I}_{3} \text { vs. } \mathrm{I}_{1}: \\
\text { SCT } \\
\text { LBT } \\
\mathrm{I}_{3} \text { vs. } \mathrm{I}_{2}: \\
\text { SCT } \\
\text { LBT } \\
\mathrm{I}_{3} \text { vs. C: } \\
\text { SCT } \\
\text { LBT }\end{array}$ & $\begin{array}{c}+ \\
\text { NS } \\
+ \\
+ \\
\text { NS } \\
+ \\
+ \\
+\end{array}$ & $\begin{array}{l}\text { NS } \\
\text { NS } \\
+ \\
+ \\
+ \\
+ \\
+ \\
\end{array}$ & $\begin{array}{l}4 \text { weeks after last } \\
\text { cTBS session }\end{array}$ \\
\hline
\end{tabular}

Note: column 2 provides the different groups included in the corresponding studies, column 7 provides outcome measures used to compare these groups, columns 8 and 9 provide the results of between-group analyses. F5, P3 and P5 are positions based on the $10-20$ EEG system. Abbreviations: AMT = active motor threshold; BCT = Bird Cancellation Test; BG= between-groups; $\mathrm{BIT}=$ Behavioral Inattention Test $C=$ control group $\mathrm{CBS}=$ Catherine Bergego Scale CORCT = crossover randomized controlled trial; $\mathrm{CT}=$ concurrent therapy; FIM = Functional Independence Measure; $I=$ intervention group; LBT = line bisection test; LIMOS = Lucerne ICF-based Multidisciplinary Observation Scale; MFT = movement function training; MRS = Modified Rankin Scale; MRT = Munich Reading Texts; N/A=not available; $\mathrm{PPC}=$ posterior parietal cortex; RCT = randomized controlled trial; RMT = resting motor threshold; RSCT $=$ Random Shape Cancellation Test; $\mathrm{SCT}=$ star cancellation test; TPPT $=$ Two Part Picture Test; VTS $=$ Vienna test system; $+=$ significant at $p<0.05$. 
Table 6

Summary of the best evidence synthesis

\begin{tabular}{|c|c|c|c|c|c|c|}
\hline \multirow[t]{2}{*}{ Effectiveness of } & \multirow[t]{2}{*}{ ICF-category } & \multicolumn{4}{|c|}{$\begin{array}{l}\text { Number of high-quality } \\
\text { RCTs }\end{array}$} & \multirow[t]{2}{*}{ Level of evidence } \\
\hline & & Total & $\begin{array}{l}\text { Favor } \\
\text { cTBS }\end{array}$ & $\begin{array}{l}\text { No sig } \\
\text { diff. }\end{array}$ & $\begin{array}{l}\text { Favor } \\
\text { control }\end{array}$ & \\
\hline $\begin{array}{l}\text { cTBS over the left contralesional PPC on neglect } \\
\text { severity, immediately after last session }\end{array}$ & $\begin{array}{l}\text { Health condition } \\
\quad \text { (disorder or disease) }\end{array}$ & 8 & 7 & 1 & 0 & $\begin{array}{l}\text { Strong evidence in } \\
\text { favor of cTBS }\end{array}$ \\
\hline $\begin{array}{l}\text { cTBS over the left contralesional PPC on neglect } \\
\text { severity, at least two weeks after last session }\end{array}$ & $\begin{array}{l}\text { Health condition } \\
\quad \text { (disorder or disease) }\end{array}$ & 5 & 5 & 0 & 0 & $\begin{array}{l}\text { Strong evidence in } \\
\text { favor of cTBS }\end{array}$ \\
\hline $\begin{array}{l}\text { cTBS over the left contralesional PPC on ADL in } \\
\text { neglect patients, immediately after last session }\end{array}$ & Activity (limitations) & 3 & 2 & 1 & 0 & $\begin{array}{l}\text { Strong evidence in } \\
\text { favor of cTBS }\end{array}$ \\
\hline $\begin{array}{l}\text { cTBS over the left contralesional PPC on ADL in } \\
\text { neglect patients, at least two weeks after last session }\end{array}$ & Activity (limitations) & 2 & 2 & 0 & 0 & $\begin{array}{l}\text { Strong evidence in } \\
\text { favor of cTBS }\end{array}$ \\
\hline $\begin{array}{l}\text { iTBS over the left contralesional DLPFC on neglect } \\
\text { severity in neglect patients, immediately after last } \\
\text { session }\end{array}$ & $\begin{array}{l}\text { Health condition } \\
\quad \text { (disorder or disease) }\end{array}$ & 1 & 1 & 0 & 0 & $\begin{array}{l}\text { Limited evidence } \\
\text { in favor of iTBS }\end{array}$ \\
\hline
\end{tabular}

Note: The results of the best evidence synthesis were divided in different categories based on the following study characteristics: intervention protocol, location of stimulation, outcome measures, and time of measurement. Abbreviations: $\mathrm{ADL}=$ activities of daily living; cTBS = continuous theta-burst stimulation; DLPFC $=$ dorsolateral prefrontal cortex; iTBS $=$ intermittent theta-burst stimulation; $\mathrm{PPC}=$ posterior parietal cortex.

\subsection{Adverse events}

Six out of the nine included studies reported on the absence or presence of adverse events (Cazzoli et al., 2012; Fu et al., 2015, 2017; Koch et al., 2012; Nyffeler et al., 2019; Vatanparasti et al., 2019). No serious side-effects (such as severe pain, vertigo, seizures or paraesthesia) were reported in any of these studies. Fu and colleagues (Fu et al., 2015) did report that two patients experienced a slight headache after TBS, but indicated an absence of severe adverse events. Three studies did not report information about the occurrence of adverse events (Cao et al., 2016; Hopfner et al., 2015; Yang et al., 2015).

\subsection{Effectiveness of cTBS on neglect severity}

\subsubsection{Immediate effects}

A summary of the BES is provided in Table 6. Eight of the included studies evaluated the effects of cTBS on neglect symptoms. All of these studies used diagnostic instruments as one of their outcome measures, that were classified as measures of neglect severity.

Seven out of the eight studies found significant between-group differences in neglect amelioration in favor of cTBS when compared to either sham cTBS, low-intensity cTBS, or concurrent therapy without cTBS (Cao et al., 2016; Cazzoli et al., 2012; Fu et al., 2015, 2017; Hopfner et al., 2015; Koch et al., 2012; Nyffeler et al., 2019; Yang et al., 2015). One study found no significant between-group differences (Vatanparasti et al., 2019). See Table 5 for further details on between-group results. Importantly however, no statistically significant negative results were found. This means that the available evidence is highly consistent. As the number of studies that show evidence is $>50 \%$ of the total number of studies found within this category, and the results do not conflict with one another, it can be concluded that there is strong evidence for the effectiveness of cTBS over the left contralesional PPC on amelioration of neglect severity immediately after therapy, based on the criteria set out by van Tulder et al. (1999) and van Peppen et al. (2004).

\subsubsection{Effects at follow-up}

Five studies assessing the effectiveness of cTBS on neglect severity performed measures after a followup period (Cazzoli et al., 2012; Fu et al., 2015; Koch et al., 2012; Nyffeler et al., 2019; Yang et al., 2015). The follow-up periods ranged from 2 weeks to 3 months after the last cTBS session. All five studies found significant between-group differences in neglect amelioration in favor of cTBS when compared to either sham cTBS or concurrent therapy without cTBS. This indicates that there is strong evidence for the effectiveness of cTBS over the left contralesional PPC on amelioration of neglect severity, as measured by diagnostic instruments, up to at least 2 weeks after the last cTBS session. 


\subsection{Effectiveness of cTBS on activities of daily living}

\subsubsection{Immediate effects}

Three studies (Cazzoli et al., 2012; Nyffeler et al., 2019; Vatanparasti et al., 2019) that evaluated cTBS used outcome measures that assess ADL. These outcome measures were the CBS (Cazzoli et al., 2012; Nyffeler et al., 2019), the FIM (Nyffeler et al., 2019), the LIMOS (Nyffeler et al., 2019), and the MRS (Vatanparasti et al., 2019). Two studies (Cazzoli et al., 2012; Nyffeler et al., 2019) found significant between-group differences in amelioration of ADL in favor of neglect patients who received cTBS when compared to those who received either sham cTBS or concurrent therapy without cTBS. One study (Vatanparasti et al., 2019) found no such significant difference. Based on the BES criteria, it can be concluded that there is strong evidence for the effectiveness of cTBS over the left contralesional PPC on amelioration of ADL in neglect patients, immediately after therapy.

\subsubsection{Effects at follow-up}

Two studies (Cazzoli et al., 2012; Nyffeler et al., 2019) that used measures assessing ADL performed measures after a follow-up period. The follow-up periods were 2 weeks (Cazzoli et al., 2012) and 3 months (Nyffeler et al., 2019) after the last cTBS session. Both studies found significant between-group differences in amelioration of ADL in neglect patients in favor of cTBS when compared to sham cTBS or concurrent therapy without cTBS, indicating strong evidence for effectiveness up to at least 2 weeks after the last cTBS session.

\subsection{Effectiveness of iTBS on neglect severity}

One study evaluated the effects of iTBS on neglect symptoms, and used diagnostic instruments as outcome measures (Cao et al., 2016). This study found significant between-group differences in neglect amelioration in favor of iTBS when compared to low-intensity iTBS. Since only one high-quality RCT assessed iTBS, it can be concluded that there is limited evidence for the effectiveness of iTBS over the left contralesional DLPFC on amelioration of neglect severity, as measured by diagnostic instruments, immediately after therapy.

\section{Discussion}

The main objective of the present study was to systematically review the available literature (RCTs only) and investigate whether TBS of the contralesional hemisphere is effective in improving symptoms of unilateral neglect in patients with right hemisphere stroke. Nine RCTs were included in this review, of which eight evaluated the effectiveness of cTBS and one evaluated the effectiveness of iTBS. The results of these RCTs allow the conclusion that TBS can have favorable effects on amelioration of stroke-induced UN, despite heterogeneity among the included studies regarding diagnostic assessments, treatment protocols, concurrent therapies, number of therapy sessions, treatment duration, and outcome measures.

\subsection{Theoretical evaluation}

The findings of this systematic review support the notion that inhibitory NIBS, by means of cTBS, of the left contralesional hemisphere can result in significant UN symptom reduction in right-hemispheric stroke patients. These findings are in line with the interhemispheric rivalry model originally put forward by Kinsbourne $(1970,1977)$, which postulates that right side brain damage will lead to a hypoactive ipsilesional hemisphere and a hyperactive contralesional hemisphere. This is thought to induce an imbalance within a competitive attentional network, resulting in UN. Reducing the hyperexcitability of the left contralesional hemisphere could facilitate functional recovery from $\mathrm{UN}$, by rebalancing the mutual interhemispheric transcallosal inhibition.

Koch et al. (Koch et al., 2012), who measured how cTBS modified the excitability of the parietofrontal functional connections in the undamaged left hemisphere, indeed found that hyperexcitability of the left hemisphere was reduced following cTBS but not after sham stimulation. They also found that this reduction in hyperexcitability of the contralesional hemisphere led to amelioration of UN symptoms, thus pointing to the importance of rebalancing hemispheric activity. Despite these promising findings, recent studies also indicate that the contralesional hemisphere could have a compensatory role in some stroke patients (Lunven et al., 2015; Umarova et al., 2016), suggesting that activity of the contralesional hemisphere should be facilitated rather than inhibited. For instance, Cao et al. (Cao et al., 2016) found that increasing the activity of the left contralesional 
DLPFC through iTBS can facilitate recovery from UN. If the contralesional hemisphere does indeed have a compensatory role, inhibitory stimulation of this hemisphere could thus be detrimental to UN recovery. Nyfeller and colleagues (2019) aimed to address this controversial issue. They suggested that the hyperexcitability of the contralesional PPC can be seen as a loss of functional connectivity, and that cTBS cannot only reduce the over-excitability of the contralesional PPC, but may also functionally reintegrate the left PPC into attentional networks. The functional role of the contralesional PPC in attentional processes may thus be reinstated by means of inhibitory cTBS. They hypothesised that this may be the primary mechanism behind UN recovery through cTBS, and that this may be the reason why inhibition of the contralesional PPC did not cause worsening UN symptoms in any patient in their study (Nyffeler et al., 2019).

Note also that UN may originate from disfunction in several cortical areas and attentional networks (Koch et al., 2011), which could indicate that the specific site of stimulation over the contralesional hemisphere is a crucial factor in determining whether excitatory or inhibitory TBS would be beneficial for UN treatment. This idea is emphasized by functional-anatomical attention network models for UN (Baldassarre et al., 2014; Koch et al., 2011).

Although there is converging evidence about which brain areas and attentional networks are related to UN symptoms, it is clear that the underlying dysfunctions of UN are not yet sufficiently understood. Future neuroimaging research (e.g., studies using magnetic resonance imaging, electroencephalography, or novel imaging and stimulation techniques) should be conducted to investigate the mechanisms of NIBS intervention in relation to stroke-induced UN. Knowledge about whether therapeutic strategies properly target the underlying dysfunctions of UN may have a great impact on therapy and rehabilitation outcome.

\subsection{Patient suitability for TBS therapy}

Patient characteristics may play a crucial role in the effectiveness of TBS therapy on UN. Nyfeller et al. (2019) aimed to identify patient characteristics that could distinguish cTBS responders from cTBS non-responders. They found that none of various clinical and demographic parameters considered (i.e. sex, age, years of education, time since onset of stroke, lesion volume, handedness, Montreal
Cognitive Assessment score, and NIH Stroke Scale score) could predict which patients would respond to the intervention (Nyffeler et al., 2019). However, they found that cTBS non-responders presented with a lesion in the posterior part of the corpus callosum, that contains transcallosal inhibitory projections that interconnect the PPC's of the two hemispheres (Nyffeler et al., 2019). They also found that initial neglect severity significantly correlated with recovery from UN symptoms in cTBS responders but not in nonresponders. Stronger improvements of UN symptoms after TBS therapy have been reported in patients who are more severely affected (Koch et al., 2012). Therefore, it is possible that more pronounced and clinically relevant effects will be found when TBS is applied to more severely affected patients. Nonetheless, as side effects are rare and worsening of UN symptoms after TBS was not reported in any of the included studies, the risk-benefit ratio of TBS therapy seems to be favorable also in more mildly affected patients.

Another potentially crucial patient characteristic may be the specific subtype of UN the patient has. Attempts have been made to define UN subtypes (and their neurological correlates), along various dimensions such as which modality (i.e. sensory, motor or representational) and distribution of attention in space (i.e. personal, peripersonal or extrapersonal space) is affected (Heilman et al., 2000; Kerkhoff, 2001; Plummer et al., 2003). There is evidence to support the notion that patients with certain UN symptom profiles (i.e. certain UN subtypes) respond differently to specific treatments (Barrett et al., 2006). Even though various classification systems have been proposed (Heilman et al., 2000; Plummer et al., 2003; Rode et al., 2017), this is not always taken into account in the literature. The heterogeneity of UN subtypes might account for the high variability in effectiveness of NIBS protocols (Lefaucheur et al., 2014), as patients with different UN subtypes may respond differently to treatment (Barrett et al., 2006). As a case in point, Spaccavento et al. (2017) reported differences in functional rehabilitation outcome between patients with personal, peripersonal and extrapersonal neglect who received cognitive, physical and occupational therapy. Whether UN subtypes can explain some of the variability in effectiveness of therapy with NIBS needs to be further investigated. Interestingly, the outcome measures assessing neglect severity that were used in the included studies in this review predominantly involve neglect in peripersonal space, so the results of this 
review seem to be most applicable to patients with this UN subtype. Improvement of UN classification and diagnostic instruments could potentially be of great value in determining the effectiveness of TBS for the treatment of specific UN subtypes, and could therefore hold the promise for more tailor-made forms of UN treatment.

Concerning the safety of TBS therapy, none of the included studies reported severe adverse events. Three (Cazzoli et al., 2012; Fu et al., 2015; Nyffeler et al., 2019) out of the nine included studies reported to have followed the internationally accepted safety guidelines for the application of TMS (Rossi et al., 2009), and four (Cao et al., 2016; Fu et al., 2017; Vatanparasti et al., 2019; Yang et al., 2015) out of the nine included studies reported to have excluded patients with contraindications for TMS. It therefore seems to be important to follow these safety guidelines (Rossi et al., 2009) when applying TBS, to ensure patient safety.

\subsection{TBS protocols for clinical use}

TBS intervention protocols and the number of TBS sessions varied across the included studies. Different types of protocols thus produced favorable effects on UN symptoms. Looking at the included RCTs, the following cTBS protocol could be recommended for clinical use: a cTBS protocol of uninterrupted trains of 3-pulse 30 or $50 \mathrm{~Hz}$ bursts with a 5 or $6 \mathrm{~Hz}$ inter-burst frequency, administered at 80 or $100 \%$ RMT. The only iTBS protocol included in this review consisted of 20 trains of 3-pulse $50 \mathrm{~Hz}$ bursts, repeated at $5 \mathrm{~Hz}$, with an inter-train interval of $8 \mathrm{sec}$, at $80 \%$ RMT. As the cTBS protocol in the study from Vatanparasti et al. (2019) was the only one not to produce a significantly greater improvement of UN symptoms, cTBS protocols with a $10 \mathrm{~Hz}$ interburst frequency are not recommended for clinical use, based on the included RCTs. It is not certain, however, that this higher than normal inter-burst frequency is the primary cause of the non-significant findings, as Vatanparasti et al. (2019) also had a deviating study population (i.e. also chronic stroke patients) and used a concurrent therapy that no other included study used (i.e. prism adaptation). TBS protocols with different stimulation parameters (e.g. protocols with a $30 \mathrm{~Hz}$ or $50 \mathrm{~Hz}$ inter-pulse frequencies) do seem to elicit different neuroplastic responses (Goldsworthy et al., 2012); at least, when applied to the primary motor cortex. Non-optimal stimulation characteristics could possibly be an explanation for the high variability in effectiveness of TBS therapy. Indeed, different TBS protocols should be directly compared in future research to investigate the optimal stimulation parameters for clinical UN treatment.

A recommendation concerning the optimal number of TBS sessions for UN treatment is not possible given the state of the current literature, as the studies varied greatly in treatment duration and the number of TBS sessions per day. It is uncertain whether the efficacy of TBS can be further enhanced by increasing the treatment duration (i.e. adding more consecutive days of TBS therapy) or by increasing the number of TBS trains administered per day. It does seem to be the case that increasing the number of applied TBS sessions leads to a better outcome, as there is evidence that increasing the number TBS sessions leads to a disproportional increase in after-effects, possibly through long term depression- and potentiation-like mechanisms that can be consolidated by the repeated application of TBS (Goldsworthy et al., 2015). For instance, in UN patients, it has been shown that four trains of cTBS have considerably stronger effects than two trains of cTBS (Nyffeler et al., 2009). Nevertheless, as reported by Nyfeller et al. (2019), both 8 sessions and 16 sessions of cTBS provided similar improvement of UN symptoms, at least when combined with SPT. This suggests that there is an upper limit to the number of sessions required for TBS intervention to have favorable effects on UN recovery. A possible explanation for this 'ceiling effect', in the case of inhibitory cTBS, is that a certain number of cTBS trains would already have reduced the over-excitability of the contralesional hemisphere to a sufficiently low degree, and further stimulation may be resisted by physiological activity due to consolidated synaptic plasticity (Goldsworthy et al., 2015; Nyffeler et al., 2019). Future research should be conducted to investigate the optimal number of TBS sessions for UN treatment, and whether treatment duration should be extended or that the number of trains per day should be increased to enhance the effectiveness of TBS therapy.

\subsection{Combining therapeutic interventions}

In the included RCTs, many forms of concurrent therapies were used. Theoretically, combining different therapeutic interventions can result in one of three things: further improvement in effectiveness, a deterioration in effectiveness, or no distinguishable additional effects, when compared to only applying one of the therapies (Hopfner et al., 2015). 
Looking at the results of the included RCTs, it seems to be the case that TBS improves the effectiveness of several conventional neglect therapies, such as visual scanning training and smooth pursuit eye-movement training. Only when comparing the combination of cTBS and prism adaptation with prism adaptation alone, no significant between-group differences were found (Vatanparasti et al., 2019). Once again, it is not certain that this study characteristic alone is the definitive reason for the non-significant results, as this study also had an atypical cTBS protocol and study population (Vatanparasti et al., 2019).

It is not well understood why some treatment combinations may provide additive or synergistic effects, while others may not (Hopfner et al., 2015). It has been hypothesized that different therapies have effects on different brain structures and influence the attentional networks in different ways (Hopfner et al., 2015). Some therapeutic combinations could therefore have additive effects because the individual treatments activate different brain areas and attentional networks. Yet, other combinations may not have such additive effects as the individual interventions activate similar areas. The identification of suitable combinations of different therapeutic interventions for $\mathrm{UN}$ treatment is an important challenge for future research and clinical UN treatment.

\subsection{Limitations}

The available literature has several limitations. Some of the included studies (Cazzoli et al., 2012; Koch et al., 2012; Nyffeler et al., 2019) described their study design as 'double-blind', which could refer to the blinding of the participants and the experimenters. Yet, if not clearly defined, this is left ambiguous. Studies should therefore clearly define who is blinded to the intervention and group allocation. Also, allocation concealment was described in only one of the included RCTs (Nyffeler et al., 2019), while this could relatively easily be implemented to increase methodological quality and decrease risk of bias. Furthermore, several studies indicated blinding of the therapists but did not describe how this blinding was performed (Fu et al., 2015; Koch et al., 2012). Blinding therapists who perform sham or lowintensity TBS is not easily done. Studies should therefore elaborate on the blinding process of their administering therapists. In future research, these methodological aspects should be more carefully considered and reported.
The present review also has several limitations that need to be acknowledged. Firstly, some subjectivity is introduced in a systematic review when performing a BES, as the criteria for the different levels of evidence are set out in a relatively arbitrary manner (van Peppen et al., 2004; van Tulder et al., 1999). A more quantitative, rather than qualitative, analysis of the data, such as a meta-analysis, is therefore in order, assuming that this is possible given the heterogeneity among the available studies. Second, no effort has been made to find unpublished papers or articles written in languages other than English. And lastly, the presence of a potential publication bias has not been statistically assessed. In some cases, publication bias can be demonstrated using funnel plots, but that is beyond the scope of this review.

\subsection{Future research}

Some recommendations concerning future research have already been made in the previous sections of this review. To summarize, investigating the following topics may enhance the effectiveness and understanding of TBS therapy on UN symptoms: (1) underlying mechanisms of TBS intervention over different brain areas in relation to stroke-induced $\mathrm{UN}$; (2) improvement of UN classification and diagnostic instruments; (3) a direct comparison of different TBS protocols; (4) a direct comparison between the effects of an increase in the number of TBS sessions and the effects of an increase in treatment duration; and (5) a direct comparison of different combinations of therapeutic interventions.

Studying the underlying mechanisms of TBS intervention with neuroimaging studies may shed light on whether therapeutic strategies properly target the underlying dysfunctions of UN. This could also be important in determining suitable combinations of different therapeutic options for UN treatment. As the lack of clear distinctions between UN subtypes might explain the high variability in effectiveness of NIBS protocols, improving UN classification and diagnostic instruments may have a great impact on clinical UN treatment. It could be the case that only patients with certain UN subtypes respond well to TBS therapy. In the current review, different TBS protocols have been shown to have favorable effects on UN symptoms, especially as regards attention in peripersonal space. Different TBS protocols with varying stimulation parameters (i.e. protocols with different inter-pulse frequencies, inter-burst frequencies and total number of pulses delivered) should be 
compared directly to investigate which protocol leads to the most optimal clinical results. As it is uncertain whether the efficacy of TBS can be further enhanced by increasing the treatment duration or by increasing the number of TBS trains administered per day, these intervention characteristics should also be compared. It is also important to know at what point administration of even more TBS sessions fails to be beneficial, in order to prevent medically unnecessary sessions. Lastly, to optimize clinical UN treatment, different combinations of therapeutic interventions should be directly compared to investigate which combination results in the most amelioration of UN symptoms.

Future research needs to be conducted using large sample sizes, consistent and standardized diagnostic assessment tools and outcome measures, uniform and consensus-based terminology, rigorous methodological quality and design, and detailed reporting of methods and findings. Preregistration of future trials can increase transparency and quality of evidence, as this will decrease the possibility for researchers to (unintentionally) bias their results.

\section{Conclusions}

In conclusion, there is evidence that TBS can have beneficial therapeutic effects on UN symptoms in stroke patients. There is strong evidence that cTBS over the left contralesional PPC is effective for the treatment of neglect severity and UN related problems in ADL, immediately after therapy, and up to at least two weeks after the last therapy session. In addition, there is limited evidence that iTBS over the left contralesional DLPFC is effective for the treatment of neglect severity immediately after therapy. Using TBS intervention for clinical treatment of stroke-induced UN can therefore be recommended.

TBS therapy seems to be more effective in patients with more severe symptoms, and in patients who have an intact corpus callosum. Patient safety is naturally of great importance. Thus, internationally accepted safety guidelines for the application of TMS should be followed to ensure that the patients have little risk of severe adverse events (Rossi et al., 2009). Still, as side effects and worsening of UN are uncommon when administering TBS therapy, the risk-benefit ratio seems to be favorable. It is recommended to use clinical TBS therapy in conjunction with cognitive rehabilitation (e.g. visual scanning training and smooth pursuit eye-movement training) and occupational or physical rehabilitation as needed.
The results of this review suggest that there is a promising future for the treatment of UN, one of the most disabling post-stroke disorders. The favorable effects of TBS may be further enhanced as more studies are performed in this field of research.

\section{Acknowledgments}

The authors declare that they have no competing interest. This research did not receive any specific grant from funding agencies in the public, commercial, or not-for-profit sectors.

\section{Supplementary material}

The supplementary material provides the full search strings for the PubMed, Ovid and Cochrane Library electronic database search.

\section{References}

Baldassarre, A., Ramsey, L., Hacker, C.L., Callejas, A., Astafiev, S.V., Metcalf, N.V., Zinn, K., Rengachary, J., Snyder, A.Z., Carter, A.R., Shulman, G.L., \& Corbetta, M. (2014). Large-scale changes in network interactions as a physiological signature of spatial neglect. Brain : A Journal of Neurology, 137(Pt 12), 3267-3283. https://doi.org/10.1093/ brain/awu297

Barrett, A.M., Buxbaum, L.J., Coslett, H.B., Edwards, E., Heilman, K.M., Hillis, A.E., Milberg, W.P., \& Robertson, I.H. (2006). Cognitive rehabilitation interventions for neglect and related disorders: moving from bench to bedside in stroke patients. Journal of Cognitive Neuroscience, 18(7), 1223-1236. https://doi.org/10.1162/jocn.2006.18.7.1223

Bernhardt, J., Hayward, K.S., Kwakkel, G., Ward, N.S., Wolf, S.L., Borschmann, K., Krakauer, J.W., Boyd, L.A., Carmichael, S.T., Corbett, D., \& Cramer, S.C. (2017). Agreed definitions and a shared vision for new standards in stroke recovery research: The Stroke Recovery and Rehabilitation Roundtable taskforce. International Journal of Stroke : Official Journal of the International Stroke Society, 12(5), 444-450. https:// doi.org/10.1177/1747493017711816

Bowen, A., Hazelton, C., Pollock, A., \& Lincoln, N.B. (2013). Cognitive rehabilitation for spatial neglect following stroke. The Cochrane Database of Systematic Reviews, 2013(7), CD003586. https://doi.org/10.1002/14651858.CD003586. pub3

Buxbaum, L.J., Ferraro, M.K., Veramonti, T., Farne, A., Whyte, J., Ladavas, E., Frassinetti, F., \& Coslett, H.B. (2004). Hemispatial neglect: Subtypes, neuroanatomy, and disability. Neurology, 62(5), 749-756. https://doi.org/10.1212/ 01.wnl.0000113730.73031.f4

Campbell, B.C.V., \& Khatri, P. (2020). Stroke. Lancet (London, England), 396(10244), 129-142. https://doi.org/10.1016/ S0140-6736(20)31179-X 
Cao, L., Fu, W., Zhang, Y., Huo, S., Du, J., Zhu, L., \& Song, W. (2016). Intermittent $\theta$ burst stimulation modulates restingstate functional connectivity in the attention network and promotes behavioral recovery in patients with visual spatial neglect. Neuroreport, 27(17), 1261-1265. https://doi.org/ 10.1097/WNR.0000000000000689

Cárdenas-Morales, L., Nowak, D.A., Kammer, T., Wolf, R.C., \& Schönfeldt-Lecuona, C. (2010). Mechanisms and applications of theta-burst rTMS on the human motor cortex. Brain Topography, 22(4), 294-306. https://doi.org/10.1007/ s10548-009-0084-7

Cazzoli, D., Müri, R.M., Schumacher, R., von Arx, S., Chaves, S., Gutbrod, K., Bohlhalter, S., Bauer, D., Vanbellingen, T., Bertschi, M., Kipfer, S., Rosenthal, C.R., Kennard, C., Bassetti, C.L., \& Nyffeler, T. (2012). Theta burst stimulation reduces disability during the activities of daily living in spatial neglect. Brain: A Journal of Neurology, 135(Pt 11), 3426-3439. https://doi.org/10.1093/brain/aws182

Cazzoli, D., Wurtz, P., Müri, R.M., Hess, C.W., \& Nyffeler, T. (2009). Interhemispheric balance of overt attention: a theta burst stimulation study. The European Journal of Neuroscience, 29(6), 1271-1276. https://doi.org/10.1111/ j.1460-9568.2009.06665.x

Di Monaco, M., Schintu, S., Dotta, M., Barba, S., Tappero, R., \& Gindri, P. (2011). Severity of unilateral spatial neglect is an independent predictor of functional outcome after acute inpatient rehabilitation in individuals with right hemispheric stroke. Archives of Physical Medicine and Rehabilitation, 92(8), 1250-1256. https://doi.org/10.1016/ j.apmr.2011.03.018

Di Pino, G., Pellegrino, G., Assenza, G., Capone, F., Ferreri, F., Formica, D., Ranieri, F., Tombini, M., Ziemann, U., Rothwell, J.C., \& Di Lazzaro, V. (2014). Modulation of brain plasticity in stroke: a novel model for neurorehabilitation. Nature Reviews. Neurology, 10(10), 597-608. https://doi.org/ 10.1038/nrneurol.2014.162

Esposito, E., Shekhtman, G., \& Chen, P. (2021). Prevalence of spatial neglect post-stroke: A systematic review. Annals of Physical and Rehabilitation Medicine, 64(5), 101459. https:// doi.org/10.1016/j.rehab.2020.10.010

Feigin, V.L., Roth, G.A., Naghavi, M., Parmar, P., Krishnamurthi, R., Chugh, S., Mensah, G.A., Norrving, B., Shiue, I., Ng, M., Estep, K., Cercy, K., Murray, C.J.L., \& Forouzanfar, M.H. (2016). Global burden of stroke and risk factors in 188 countries, during 1990-2013: a systematic analysis for the Global Burden of Disease Study 2013. The Lancet. Neurology, 15(9), 913-924. https://doi.org/10.1016/S1474-4422(16)30073-4

Fu, W., Cao, L., Zhang, Y., Huo, S., Du, J., Zhu, L., \& Song, W. (2017). Continuous theta-burst stimulation may improve visuospatial neglect via modulating the attention network: a randomized controlled study. Topics in Stroke Rehabilitation, 24(4), 236-241. https://doi.org/10.1080/10749357. 2016.1253139

Fu, W., Song, W., Zhang, Y., Yang, Y., Huo, S., Zhang, R., \& Wang, M. (2015). Long-term effects of continuous theta-burst stimulation in visuospatial neglect. The Journal of International Medical Research, 43(2), 196-203. https://doi.org/10.1177/ 0300060513498663

Gillen, R., Tennen, H., \& McKee, T. (2005). Unilateral spatial neglect: relation to rehabilitation outcomes in patients with right hemisphere stroke. Archives of Physical Medicine and Rehabilitation, 86(4), 763-767. https://doi.org/10.1016/ j.apmr.2004.10.029

Goldsworthy, M.R., Müller-Dahlhaus, F., Ridding, M.C., \& Ziemann, U. (2015). Resistant Against De-depression: LTD-Like Plasticity in the Human Motor Cortex Induced by Spaced cTBS. Cerebral Cortex (New York, N.Y. : 1991), 25(7), 1724-1734. https://doi.org/10.1093/cercor/bht353

Goldsworthy, M.R., Pitcher, J.B., \& Ridding, M.C. (2012). A comparison of two different continuous theta burst stimulation paradigms applied to the human primary motor cortex. Clinical Neurophysiology : Official Journal of the International Federation of Clinical Neurophysiology, 123(11), 2256-2263. https://doi.org/10.1016/j.clinph.2012.05.001

Heilman, K.M., Valenstein, E., \& Watson, R.T. (2000). Neglect and related disorders. Seminars in Neurology, 20(4), 463-470. https://doi.org/10.1055/s-2000-13179

Hopfner, S., Cazzoli, D., Müri, R.M., Nef, T., Mosimann, U.P., Bohlhalter, S., Vanbellingen, T., \& Nyffeler, T. (2015). Enhancing treatment effects by combining continuous theta burst stimulation with smooth pursuit training. Neuropsychologia, 74, 145-151. https://doi.org/10.1016/j.neuropsy chologia.2014.10.018

Huang, Y.-Z., Edwards, M.J., Rounis, E., Bhatia, K.P., \& Rothwell, J.C. (2005). Theta burst stimulation of the human motor cortex. Neuron, 45(2), 201-206. https://doi.org/10.1016/ j.neuron.2004.12.033

Karnath, H.-O., Rennig, J., Johannsen, L., \& Rorden, C. (2011). The anatomy underlying acute versus chronic spatial neglect: a longitudinal study. Brain : A Journal of Neurology, 134 (Pt 3), 903-912. https://doi.org/10.1093/brain/awq355

Kerkhoff, G. (2001). Spatial hemineglect in humans. Progress in Neurobiology, 63(1), 1-27. https://doi.org/10.1016/s03010082(00)00028-9

Kinsbourne, M. (1970). A model for the mechanism of unilateral neglect of space. Transactions of the American Neurological Association, 95, 143-146.

Kinsbourne, M. (1977). Hemi-neglect and hemisphere rivalry. Advances in Neurology, 18, 41-49.

Koch, G., Bonnì, S., Giacobbe, V., Bucchi, G., Basile, B., Lupo, F., Versace, V., Bozzali, M., \& Caltagirone, C. (2012). $\theta$-burst stimulation of the left hemisphere accelerates recovery of hemispatial neglect. Neurology, 78(1), 24-30. https://doi.org/ 10.1212/WNL.0b013e31823ed08f

Koch, G., Cercignani, M., Bonni, S., Giacobbe, V., Bucchi, G., Versace, V., Caltagirone, C., \& Bozzali, M. (2011). Asymmetry of parietal interhemispheric connections in humans. The Journal of Neuroscience : The Official Journal of the Society for Neuroscience, 31(24), 8967-8975. https://doi.org/10.1523/ JNEUROSCI.6567-10.2011

Langhorne, P., Bernhardt, J., \& Kwakkel, G. (2011). Stroke rehabilitation. Lancet (London, England), 377(9778), 1693-1702. https://doi.org/10.1016/S0140-6736(11)60325-5

Lefaucheur, J.-P., André-Obadia, N., Antal, A., Ayache, S.S., Baeken, C., Benninger, D.H., Cantello, R.M., Cincotta, M., de Carvalho, M., De Ridder, D., Devanne, H., Di Lazzaro, V., Filipović, S.R., Hummel, F.C., Jääskeläinen, S.K., Kimiskidis, V.K., Koch, G., Langguth, B., Nyffeler, T., ... Garcia-Larrea, L. (2014). Evidence-based guidelines on the therapeutic use of repetitive transcranial magnetic stimulation (rTMS). Clinical Neurophysiology : Official Journal of the International 
Federation of Clinical Neurophysiology, 125(11), 21502206. https://doi.org/10.1016/j.clinph.2014.05.021

Lunven, M., Thiebaut De Schotten, M., Bourlon, C., Duret, C., Migliaccio, R., Rode, G., \& Bartolomeo, P. (2015). White matter lesional predictors of chronic visual neglect: a longitudinal study. Brain : A Journal of Neurology, 138(Pt 3), 746-760. https://doi.org/10.1093/brain/awu389

Maaijwee, N.A.M.M., Rutten-Jacobs, L.C.A., Arntz, R.M., Schaapsmeerders, P., Schoonderwaldt, H.C., van Dijk, E.J., $\&$ de Leeuw, F.-E. (2014). Long-term increased risk of unemployment after young stroke: a long-term follow-up study. Neurology, 83(13), 1132-1138. https://doi.org/10.1212/ WNL.0000000000000817

Moher, D., Liberati, A., Tetzlaff, J., \& Altman, D.G. (2009). Preferred reporting items for systematic reviews and metaanalyses: the PRISMA statement. PLoS Medicine, 6(7), e1000097. https://doi.org/10.1371/journal.pmed.1000097

Moseley, A.M., Herbert, R.D., Sherrington, C., \& Maher, C.G. (2002). Evidence for physiotherapy practice: a survey of the Physiotherapy Evidence Database (PEDro). The Australian Journal of Physiotherapy, 48(1), 43-49. https://doi.org/ 10.1016/s0004-9514(14)60281-6

Nyffeler, T., Cazzoli, D., Hess, C.W., \& Müri, R.M. (2009). One session of repeated parietal theta burst stimulation trains induces long-lasting improvement of visual neglect. Stroke, 40(8), 2791-2796. https://doi.org/10.1161/STROKEAHA. 109.552323

Nyffeler, T., Vanbellingen, T., Kaufmann, B.C., Pflugshaupt, T., Bauer, D., Frey, J., Chechlacz, M., Bohlhalter, S., Müri, R.M., Nef, T., \& Cazzoli, D. (2019). Theta burst stimulation in neglect after stroke: functional outcome and response variability origins. Brain : A Journal of Neurology, 142(4), 992-1008. https://doi.org/10.1093/brain/awz029

Nyffeler, T., Wurtz, P., Lüscher, H.-R., Hess, C.W., Senn, W., Pflugshaupt, T., von Wartburg, R., Lüthi, M., \& Müri, R.M. (2006). Extending lifetime of plastic changes in the human brain. The European Journal of Neuroscience, 24(10), 2961-2966. https://doi.org/10.1111/j.1460-9568.2006. 05154.x

Oliveri, M., Bisiach, E., Brighina, F., Piazza, A., La Bua, V., Buffa, D., \& Fierro, B. (2001). rTMS of the unaffected hemisphere transiently reduces contralesional visuospatial hemineglect. Neurology, 57(7), 1338-1340. https://doi.org/ 10.1212/wnl.57.7.1338

Plummer, P., Morris, M.E., \& Dunai, J. (2003). Assessment of unilateral neglect. Physical Therapy, 83(8), 732-740.

Rode, G., Pagliari, C., Huchon, L., Rossetti, Y., \& Pisella, L. (2017). Semiology of neglect: An update. Annals of Physical and Rehabilitation Medicine, 60(3), 177-185. https://doi.org/ 10.1016/j.rehab.2016.03.003

Rossi, S., Hallett, M., Rossini, P.M., \& Pascual-Leone, A. (2009). Safety, ethical considerations, and application guidelines for the use of transcranial magnetic stimulation in clinical practice and research. Clinical Neurophysiology : Official Journal of the International Federation of Clinical Neurophysiology, 120(12), 2008-2039. https://doi.org/10.1016/ j.clinph.2009.08.016
Spaccavento, S., Cellamare, F., Falcone, R., Loverre, A., \& Nardulli, R. (2017). Effect of subtypes of neglect on functional outcome in stroke patients. Annals of Physical and Rehabilitation Medicine, 60(6), 376-381. https://doi.org/10.1016/ j.rehab.2017.07.245

Stone, S.P., Patel, P., Greenwood, R.J., \& Halligan, P.W. (1992). Measuring visual neglect in acute stroke and predicting its recovery: the visual neglect recovery index. Journal of Neurology, Neurosurgery, and Psychiatry, 55(6), 431-436. https://doi.org/10.1136/jnnp.55.6.431

Ting, D.S.J., Pollock, A., Dutton, G.N., Doubal, F.N., Ting, D.S.W., Thompson, M., \& Dhillon, B. (2011). Visual neglect following stroke: current concepts and future focus. Survey of Ophthalmology, 56(2), 114-134. https://doi.org/10.1016/ j.survophthal.2010.08.001

Umarova, R.M., Nitschke, K., Kaller, C.P., Klöppel, S., Beume, L., Mader, I., Martin, M., Hennig, J., \& Weiller, C. (2016). Predictors and signatures of recovery from neglect in acute stroke. Annals of Neurology, 79(4), 673-686. https://doi.org/ 10.1002/ana.24614

Ustün, T.B., Chatterji, S., Bickenbach, J., Kostanjsek, N., \& Schneider, M. (2003). The International Classification of Functioning, Disability and Health: a new tool for understanding disability and health. Disability and Rehabilitation, 25(11-12), 565-571. https://doi.org/10.1080/096382803 1000137063

van Peppen, R.P.S., Kwakkel, G., Wood-Dauphinee, S., Hendriks, H.J.M., Van der Wees, P.J., \& Dekker, J. (2004). The impact of physical therapy on functional outcomes after stroke: what's the evidence? Clinical Rehabilitation, 18(8), 833-862. https:// doi.org/10.1191/0269215504cr843oa

van Tulder, M.W., Cherkin, D.C., Berman, B., Lao, L., \& Koes, B.W. (1999). The effectiveness of acupuncture in the management of acute and chronic low back pain. A systematic review within the framework of the Cochrane Collaboration Back Review Group. Spine, 24(11), 1113-1123. https://doi.org/ 10.1097/00007632-199906010-00011

Vatanparasti, S., Kazemnejad, A., Yoonessi, A., \& Oveisgharan, S. (2019). The Effect of Continuous Theta-Burst Transcranial Magnetic Stimulation Combined with Prism Adaptation on the Neglect Recovery in Stroke Patients. Journal of Stroke and Cerebrovascular Diseases : The Official Journal of National Stroke Association, 28(11), 104296. https://doi.org/10.1016/ j.jstrokecerebrovasdis.2019.07.012

Wafa, H.A., Wolfe, C.D.A., Emmett, E., Roth, G.A., Johnson, C.O., \& Wang, Y. (2020). Burden of Stroke in Europe: Thirty-Year Projections of Incidence, Prevalence, Deaths, and Disability-Adjusted Life Years. Stroke, 51(8), 2418-2427. https://doi.org/10.1161/STROKEAHA.120.029606

Yang, W., Liu, T.-T., Song, X.-B., Zhang, Y., Li, Z.-H., Cui, Z.-H., Hao, Q., Liu, H.L., Lei, C.L., \& Liu, J. (2015). Comparison of different stimulation parameters of repetitive transcranial magnetic stimulation for unilateral spatial neglect in stroke patients. Journal of the Neurological Sciences, 359(1-2), 219-225. https://doi.org/10.1016/j.jns.2015.08.1541 